Document downloaded from:

http://hdl.handle.net/10251/169904

This paper must be cited as:

Boiti, C.; Jornet Casanova, D.; Oliaro, A. (2020). Real Paley-Wiener theorems in spaces of ultradifferentiable functions. Journal of Functional Analysis. 278(4):1-45.

https://doi.org/10.1016/j.jfa.2019.108348

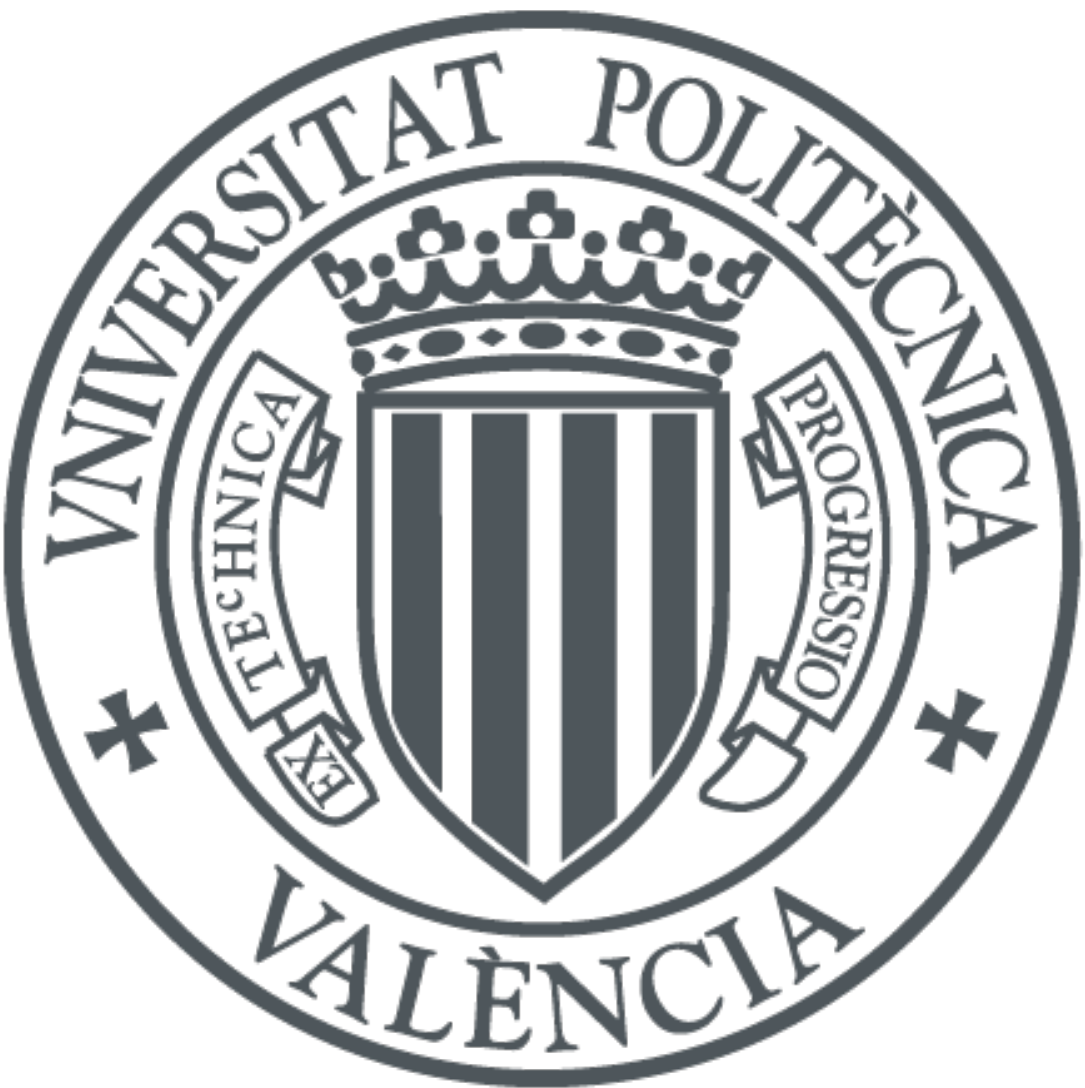

The final publication is available at

https://doi.org/10.1016/j.jfa.2019.108348

Copyright Elsevier

Additional Information 


\title{
REAL PALEY-WIENER THEOREMS IN SPACES OF ULTRADIFFERENTIABLE FUNCTIONS
}

\author{
CHIARA BOITI, DAVID JORNET, AND ALESSANDRO OLIARO
}

\begin{abstract}
We develop real Paley-Wiener theorems for classes $\mathcal{S}_{\omega}$ of ultradifferentiable functions and related $L_{p}$-spaces in the spirit of Bang and Andersen for the Schwartz class. We introduce results of this type for the so-called Gabor transform and give a full characterization in terms of Fourier and Wigner transforms for several variables of a Paley-Wiener theorem in this general setting, which is new in the literature. We also analyze this type of results when the support of the function is not compact using polynomials. Some examples are given.
\end{abstract}

\section{Introduction}

As stated in [4], "A Paley-Wiener theorem is a characterization, by relating support to growth, of the image of a space of functions or distributions under a transform of Fourier type." This relation comes only in terms of a compact and convex set in which the support of the function or distribution is included. In fact, the growth of $\hat{f}$ on $\mathbb{C}^{d}$ enables to retrieve the convex hull of the support of $f$, but no more precise information can be obtained from it (see [4] and the references therein). In the last years, a new type of results called "real Paley-Wiener type theorems" has received much attention, which try to circumvent this theoretical obstruction for the classical Paley-Wiener theorems to "look inside" the convex hull of the support. The word "real" expresses that information about the support of $f$ comes from growth rates associated to the function $\hat{f}$ on $\mathbb{R}^{d}$ rather than on $\mathbb{C}^{d}$ as in the classical "complex Paley-Wiener theorems". This theory was initiated by Bang and Tuan, and here we follow the approach of Andersen and Andersen-De Jeu (see [2, 3, 4, 5, 26] and the references therein), who state results of "real Paley-Wiener" type in spaces of rapidly decreasing functions (the Schwartz class $\mathcal{S}\left(\mathbb{R}^{d}\right)$ ) or in $L^{p}$ spaces in their most general version, using polynomials, where the support of the function (or distribution) could be non-compact or even non-convex.

Björck [6] introduced in 1966 global classes of ultradifferentiable functions $\mathcal{S}_{\omega}\left(\mathbb{R}^{d}\right)$ using weights $\omega$ in the sense of Beurling to extend previous theorems of Hörmander about interior regularity of linear partial differential operators with constant coefficients. These weight functions permit to treat in a unified way a big scale of classes of functions or (ultra)distributions and are especially suitable for manipulations on the Fourier transform side. We recall here that when the weight function is the logarithm, i.e. $\omega(t)=\log (1+t)$, the class $\mathcal{S}_{\omega}\left(\mathbb{R}^{d}\right)$ is the Schwartz class $\mathcal{S}\left(\mathbb{R}^{d}\right)$; see Definition 2.3. In the last 60 years, the classes of ultradifferentiable functions and their duals have been intensively studied for very different purposes and have

2010 Mathematics Subject Classification. Primary 46F12, Secondary 46F05, 42B10.

Key words and phrases. real Paley-Wiener theorems, weighted Schwartz classes, short-time Fourier transform, Wigner transform. 
become the right setting to study many different problems in analysis in a very general way (partial differential equations, Paley-Wiener theorems, Whitney jets, Borel theorems, etc.). We mention [14] as the reference for the modern point of view of the treatment of these classes where the authors get, under some conditions on the weight functions, to relate the growth of the functions in terms of their partial derivatives and the growth of their Fourier transforms, a property that has many advantages.

As Andersen and De Jeu mention in [4], their theorems of "real" type can be extended to other transforms of Fourier type, where the classical theorems cannot. In fact, also to more general spaces of functions as we will show below. Our aim is to study real Paley-Wiener theorems in the spirit of Bang, Andersen and Andersen and De Jeu [2, 3, 4, 5] in the more general $\mathcal{S}_{\omega}$-setting and related $L^{p}$-spaces. Moreover, we show that some transforms coming from the field of time-frequency analysis enter into the game, like the Gabor and Wigner transforms. We also study the case when the support of the Fourier transform is not necessarily compact or convex, extending some results in terms of polynomials in the spirit of $[4,26]$.

In Section 2 we give some preliminaries and definitions on weight functions, Fourier type transforms and the space $\mathcal{S}_{\omega}\left(\mathbb{R}^{d}\right)$ especially when the seminorms are given in terms of $L^{p}$-norms. In Section 3 we extend [5, Theorem 1] for several variables in the $\mathcal{S}_{\omega}$-setting in different ways (see Proposition 3.3). Also in this section we state a general version of [2, Theorem 1] for the ultradifferentiable setting and several variables (Theorem 3.2). Our main result in this section is Theorem 3.17, where we give a full characterization of the known "complex Paley-Wiener theorem" in the Beurling setting (see [14, Proposition 3.4(2)]) in terms of Wigner transforms; in this result, we assume that the support of the Fourier transform of the $\mathcal{S}_{\omega}$-function is inside a hypercube in $\mathbb{R}^{d}$. To obtain it, we need some preparation: to study the behaviour of the Gabor transform of a function $f$ in $\mathcal{S}_{\omega}\left(\mathbb{R}^{d}\right)$ with respect to a window $\psi \in \mathcal{S}_{\omega}\left(\mathbb{R}^{d}\right)$, in a suitable weighted mixed $L^{p, q}$-space, in terms of the support of the function $f$ and the window $\psi$ (Proposition 3.11). As a consequence, the symmetric properties of the Wigner transform give surprising results (Corollaries 3.14 and 3.15). We finish this section with an example about Hermite functions. In Section 4 we treat the case of arbitrary support and, following the lines of [4], we extend Theorem 2.2 and 2.5 of this paper (these are our Theorem 4.2 and Corollary 4.3). In Example 4.5 we analyze the relation of the definition of the generalized support (4.1) with the regularity of the corresponding polynomial. As an appendix we add the proof of some properties about weight functions that are useful in the paper.

\section{Preliminaries}

We begin with the definition of a non-quasianalytic weight function in the sense of [14] suitable for the Beurling case, i.e. we consider the logarithm as a non-quasianalytic weight function also.

Definition 2.1. A non-quasianalytic weight function is a continuous increasing function $\omega$ : $[0,+\infty) \rightarrow[0,+\infty)$ satisfying the following properties:

( $\alpha$ ) There exists $L \geq 1$ such that $\omega(2 t) \leq L(\omega(t)+1)$, for all $t \geq 0$;

( $\beta) \int_{1}^{+\infty} \frac{\omega(t)}{t^{2}} d t<+\infty$ 
$(\gamma)$ there exist $a \in \mathbb{R}$ and $b>0$ such that

$$
\omega(t) \geq a+b \log (1+t), \quad t \geq 0
$$

$(\delta) \varphi:[0,+\infty) \rightarrow[0,+\infty), \varphi(t):=\omega\left(e^{t}\right)$ is convex.

Then, for $\zeta \in \mathbb{C}^{d}$, we define $\omega(\zeta):=\omega(|\zeta|)$, where $|\zeta|=\sqrt{\sum_{j=1}^{d}\left|\zeta_{j}\right|^{2}}$.

Remark 2.2. We recall some well-known properties on non-quasianalytic weight functions; the proofs can be found in the literature, we recall them here for the sake of completeness.

(i) Condition ( $\alpha$ ) implies that for every $t_{1}, t_{2} \geq 0$

$$
\omega\left(t_{1}+t_{2}\right) \leq L\left(\omega\left(t_{1}\right)+\omega\left(t_{2}\right)+1\right)
$$

indeed, since $\omega$ is increasing and positive we have

$\omega\left(t_{1}+t_{2}\right) \leq \omega\left(2 \max \left\{t_{1}, t_{2}\right\}\right) \leq L\left(\omega\left(\max \left\{t_{1}, t_{2}\right\}\right)+1\right) \leq L\left(\omega\left(t_{1}\right)+\omega\left(t_{2}\right)+1\right)$.

(ii) Since (2.1) trivially implies $(\alpha)$ with $2 L$ instead of $L$, we have that $(\alpha)$ is equivalent to (2.1) (cf. [14]).

(iii) By condition $(\alpha)$ and (2.1) we easily deduce that for every $k \in \mathbb{N}$ and $t \geq 0$,

$$
\omega(k t) \leq D_{k}(\omega(t)+1)
$$

where $D_{k}=L+L^{2}+\cdots+L^{k-1}$.

(iv) By $(\beta)$ and the fact that $\omega$ is increasing, we have that $\omega(t)=o(t)$ as $t \rightarrow+\infty$ (cf. [24]). This can be deduced from the fact that

$$
\frac{\omega(t)}{t}=\int_{t}^{+\infty} \frac{\omega(t)}{s^{2}} d s \leq \int_{t}^{+\infty} \frac{\omega(s)}{s^{2}} d s .
$$

(v) By condition $(\gamma)$ we have

$$
e^{-\sigma \omega(t)} \in L^{p}\left(\mathbb{R}^{d}\right), \quad \forall \sigma \geq \frac{d+1}{b p} .
$$

Let $\omega$ be a non-quasianalytic weight and $\varphi$ as in $(\delta)$ in Definition 2.1. Then we define the Young conjugate $\varphi^{*}$ of $\varphi$ by

$$
\varphi^{*}(s):=\sup _{t \geq 0}\{t s-\varphi(t)\}, s \geq 0 .
$$

We recall that it is an increasing convex function on $[0,+\infty)$ satisfying $\varphi^{* *}=\varphi$ (see [21]).

We consider also the Fourier transform of $u \in L^{1}\left(\mathbb{R}^{d}\right)$ denoted by

$$
\mathcal{F}(u)(\xi)=\hat{u}(\xi):=\int_{\mathbb{R}^{d}} u(x) e^{-i\langle x, \xi\rangle} d x, \quad \xi \in \mathbb{R}^{d},
$$

with standard extensions to more general spaces of functions and distributions. The so-called short-time Fourier transform (or Gabor transform) of $u \in L^{2}\left(\mathbb{R}^{d}\right)$, for a window function $\psi \in$ $L^{2}\left(\mathbb{R}^{d}\right)$, is denoted by

$$
V_{\psi} u(z):=\int_{\mathbb{R}^{d}} u(y) \overline{\psi(y-x)} e^{-i\langle y, \xi\rangle} d y, \quad z=(x, \xi) \in \mathbb{R}^{2 d} .
$$


The Wigner transform of $u, v \in L^{2}\left(\mathbb{R}^{d}\right)$ is denoted by

$$
\operatorname{Wig}(u, v)(x, \xi):=\int_{\mathbb{R}^{d}} u\left(x+\frac{t}{2}\right) \overline{v\left(x-\frac{t}{2}\right)} e^{-i\langle\xi, t\rangle} d t, \quad x, \xi \in \mathbb{R}^{d} .
$$

Then we write $\operatorname{Wig} u$ for $\operatorname{Wig}(u, u)$. We refer to [18] for the classical properties of the Gabor and Wigner transforms. The setting of this work is given by the following definition.

Definition $2.3([6])$. The space $\mathcal{S}_{\omega}\left(\mathbb{R}^{d}\right)$ is the set of all $u \in L^{1}\left(\mathbb{R}^{d}\right)$ such that $u, \hat{u} \in C^{\infty}\left(\mathbb{R}^{d}\right)$ and for each $\lambda>0$ and each $\alpha \in \mathbb{N}_{0}^{d}$ we have

$$
\sup _{x \in \mathbb{R}^{d}} e^{\lambda \omega(x)}\left|D^{\alpha} u(x)\right|<+\infty \quad \text { and } \quad \sup _{\xi \in \mathbb{R}^{d}} e^{\lambda \omega(\xi)}\left|D^{\alpha} \hat{u}(\xi)\right|<+\infty .
$$

The corresponding strong dual of ultradistributions will be denoted by $\mathcal{S}_{\omega}^{\prime}\left(\mathbb{R}^{d}\right)$.

In the case that $\omega(t)=\log (1+t)$, we have $\mathcal{S}_{\omega}\left(\mathbb{R}^{d}\right)=\mathcal{S}\left(\mathbb{R}^{d}\right)$. On the other hand, for an arbitrary non-quasianalytic weight function $\omega$, by condition $(\gamma)$ of Definition 2.1 it is easy to deduce that $\mathcal{S}_{\omega}\left(\mathbb{R}^{d}\right) \subset \mathcal{S}\left(\mathbb{R}^{d}\right)$. Hence, $\mathcal{S}_{\omega}\left(\mathbb{R}^{d}\right)$ can be equivalently defined as the set of all $u \in \mathcal{S}\left(\mathbb{R}^{d}\right)$ that satisfy the condition of Definition 2.3. By Björck [6], we know that the Fourier transform $\mathcal{F}: \mathcal{S}_{\omega}\left(\mathbb{R}^{d}\right) \rightarrow \mathcal{S}_{\omega}\left(\mathbb{R}^{d}\right)$ is a continuous automorphism, that can be extended in the usual way to $\mathcal{S}_{\omega}^{\prime}\left(\mathbb{R}^{d}\right)$ when endowed with the strong topology, i.e. the topology of uniform convergence on bounded sets. Moreover, the space $\mathcal{S}_{\omega}\left(\mathbb{R}^{d}\right)$ is an algebra under multiplication and convolution. On the other hand, for $u, \psi \in \mathcal{S}_{\omega}\left(\mathbb{R}^{d}\right)$ we have $V_{\psi} u$, Wig $u \in \mathcal{S}_{\omega}\left(\mathbb{R}^{2 d}\right)$. Moreover, for $u, \psi \in \mathcal{S}_{\omega}^{\prime}\left(\mathbb{R}^{d}\right)$ the Gabor and Wigner transforms are well defined and belong to $\mathcal{S}_{\omega}^{\prime}\left(\mathbb{R}^{2 d}\right)$ $[11,12,19]$. We recall, for the convenience of the reader, the following result $[11,12]$.

Theorem 2.4. Given $u \in \mathcal{S}\left(\mathbb{R}^{d}\right)$, then $u \in \mathcal{S}_{\omega}\left(\mathbb{R}^{d}\right)$ if and only if one of the following conditions hold:

i) $\forall \lambda>0, \alpha \in \mathbb{N}_{0}^{d} \exists C_{\alpha, \lambda}>0$ s.t. $\sup _{x \in \mathbb{R}^{d}} e^{\lambda \omega(x)}\left|D^{\alpha} u(x)\right| \leq C_{\alpha, \lambda}$, and

ii) $\forall \lambda>0, \alpha \in \mathbb{N}_{0}^{d} \exists C_{\alpha, \lambda}>0$ s.t. $\sup _{\xi \in \mathbb{R}^{d}} e^{\lambda \omega(\xi)}\left|D^{\alpha} \hat{u}(\xi)\right| \leq C_{\alpha, \lambda}$;

$$
\text { i) } \forall \lambda>0, \alpha \in \mathbb{N}_{0}^{d} \exists C_{\alpha, \lambda}>0 \text { s.t. } \sup _{x \in \mathbb{R}^{d}} e^{\lambda \omega(x)}\left|x^{\alpha} u(x)\right| \leq C_{\alpha, \lambda} \text {, and }
$$

ii) $\forall \lambda>0, \alpha \in \mathbb{N}_{0}^{d} \exists C_{\alpha, \lambda}>0$ s.t. $\sup _{\xi \in \mathbb{R}^{d}} e^{\lambda \omega(\xi)}\left|\xi^{\alpha} \hat{u}(\xi)\right| \leq C_{\alpha, \lambda}$;

$$
\begin{aligned}
& \text { i) } \forall \lambda>0 \exists C_{\lambda}>0 \text { s.t. } \sup _{x \in \mathbb{R}^{d}} e^{\lambda \omega(x)}|u(x)| \leq C_{\lambda} \text {, and } \\
& \text { ii) } \forall \lambda>0 \exists C_{\lambda}>0 \text { s.t. } \sup _{\xi \in \mathbb{R}^{d}} e^{\lambda \omega(\xi)}|\hat{u}(\xi)| \leq C_{\lambda} \text {; }
\end{aligned}
$$

$$
\begin{aligned}
& \text { i) } \forall \lambda>0, \beta \in \mathbb{N}_{0}^{d} \exists C_{\beta, \lambda}>0 \text { s.t. } \sup _{\alpha \in \mathbb{N}_{0}^{d}} \sup _{x \in \mathbb{R}^{d}}\left|x^{\beta} D^{\alpha} u(x)\right| e^{-\lambda \varphi^{*}\left(\frac{|\alpha|}{\lambda}\right)} \leq C_{\beta, \lambda} \text {, and } \\
& \text { ii) } \forall \mu>0, \alpha \in \mathbb{N}_{0}^{d} \exists C_{\alpha, \mu}>0 \text { s.t. } \sup _{\beta \in \mathbb{N}_{0}^{d}} \sup _{x \in \mathbb{R}^{d}}\left|x^{\beta} D^{\alpha} u(x)\right| e^{-\mu \varphi^{*}\left(\frac{|\beta|}{\mu}\right)} \leq C_{\alpha, \mu}
\end{aligned}
$$

(e) $\forall \mu, \lambda>0 \exists C_{\mu, \lambda}>0$ s.t. $\sup _{\alpha, \beta \in \mathbb{N}_{0}^{d}} \sup _{x \in \mathbb{R}^{d}}\left|x^{\beta} D^{\alpha} u(x)\right| e^{-\lambda \varphi^{*}\left(\frac{|\alpha|}{\lambda}\right)} e^{-\mu \varphi^{*}\left(\frac{|\beta|}{\mu}\right)} \leq C_{\mu, \lambda}$;

(f) $\forall \lambda>0 \exists C_{\lambda}>0$ s.t. $\sup _{\alpha, \beta \in \mathbb{N}_{0}^{d}} \sup _{x \in \mathbb{R}^{d}}\left|x^{\beta} D^{\alpha} u(x)\right| e^{-\lambda \varphi^{*}\left(\frac{|\alpha+\beta|}{\lambda}\right)} \leq C_{\lambda}$; 
(g) $\forall \mu, \lambda>0 \exists C_{\mu, \lambda}>0$ s.t. $\sup _{\alpha \in \mathbb{N}^{d}} \sup _{x \in \mathbb{R}^{d}}\left|D^{\alpha} u(x)\right| e^{-\lambda \varphi^{*}\left(\frac{|\alpha|}{\lambda}\right)} e^{\mu \omega(x)} \leq C_{\mu, \lambda}$;

(h) given $\psi \in \mathcal{S}_{\omega}\left(\mathbb{R}^{d}\right) \backslash\{0\}, \forall \lambda>0 \exists C_{\lambda}>0$ s.t. $\sup _{z \in \mathbb{R}^{2 d}}\left|V_{\psi} u(z)\right| e^{\lambda \omega(z)} \leq C_{\lambda}$.

In the following, it is sometimes more convenient to use $L^{p}$-norms instead of $L^{\infty}$-norms in $\mathcal{S}_{\omega}\left(\mathbb{R}^{d}\right)$. We need the following notation of $L^{p, q}$-space:

$$
\begin{aligned}
L^{p, q}\left(\mathbb{R}^{2 d}\right):=\left\{F \text { measurable on } \mathbb{R}^{2 d}\right. \text { such that: } & \\
& \left.\|F\|_{L^{p, q}}:=\left(\int_{\mathbb{R}^{d}}\left(\int_{\mathbb{R}^{d}}|F(x, \xi)|^{p} d x\right)^{q / p} d \xi\right)^{1 / q}<+\infty\right\}
\end{aligned}
$$

if $1 \leq p, q<+\infty$; here, we replace the $L^{p}$ or $L^{q}$ norm with the essential supremum if $p$ or $q$ is equal to $\infty$. We obtain the next extension of Theorem 2.4:

Theorem 2.5. Given a function $u \in \mathcal{S}\left(\mathbb{R}^{d}\right)$ and $1 \leq p, q \leq+\infty$, we have that $u \in \mathcal{S}_{\omega}\left(\mathbb{R}^{d}\right)$ if and only if one of the following conditions is satisfied:

$(a)^{\prime} \quad$ i) $\forall \lambda>0, \alpha \in \mathbb{N}_{0}^{d} \exists C_{\alpha, \lambda}>0$ s.t. $\left\|e^{\lambda \omega(x)} D^{\alpha} u(x)\right\|_{L^{p}} \leq C_{\alpha, \lambda}$, and

ii) $\forall \lambda>0, \alpha \in \mathbb{N}_{0}^{d} \exists C_{\alpha, \lambda}>0$ s.t. $\left\|e^{\lambda \omega(\xi)} D^{\alpha} \hat{u}(\xi)\right\|_{L^{q}} \leq C_{\alpha, \lambda}$;

$(b)^{\prime} \quad$ i) $\forall \lambda>0, \alpha \in \mathbb{N}_{0}^{d} \exists C_{\alpha, \lambda}>0$ s.t. $\left\|e^{\lambda \omega(x)} x^{\alpha} u(x)\right\|_{L^{p}} \leq C_{\alpha, \lambda}$, and

ii) $\forall \lambda>0, \alpha \in \mathbb{N}_{0}^{d} \exists C_{\alpha, \lambda}>0$ s.t. $\left\|e^{\lambda \omega(\xi)} \xi^{\alpha} \hat{u}(\xi)\right\|_{L^{q}} \leq C_{\alpha, \lambda}$;

$(c)^{\prime}$ i) $\forall \lambda>0 \exists C_{\lambda}>0$ s.t. $\left\|e^{\lambda \omega(x)} u(x)\right\|_{L^{p}} \leq C_{\lambda}$, and

ii) $\forall \lambda>0 \exists C_{\lambda}>0$ s.t. $\left\|e^{\lambda \omega(\xi)} \hat{u}(\xi)\right\|_{L^{q}} \leq C_{\lambda}$;

$(d)^{\prime} \quad$ i) $\forall \lambda>0, \beta \in \mathbb{N}_{0}^{d} \exists C_{\beta, \lambda}>0$ s.t. $\sup \left\|x^{\beta} D^{\alpha} u(x)\right\|_{L^{p}} e^{-\lambda \varphi^{*}\left(\frac{|\alpha|}{\lambda}\right)} \leq C_{\beta, \lambda}$, and

$$
\alpha \in \mathbb{N}_{0}^{d}
$$

ii) $\forall \mu>0, \alpha \in \mathbb{N}_{0}^{d} \exists C_{\alpha, \mu}>0$ s.t. $\sup _{\beta \in \mathbb{N}_{0}^{d}}\left\|x^{\beta} D^{\alpha} u(x)\right\|_{L^{q}} e^{-\mu \varphi^{*}\left(\frac{|\beta|}{\mu}\right)} \leq C_{\alpha, \mu}$;

$(e)^{\prime} \forall \mu, \lambda>0 \exists C_{\mu, \lambda}>0$ s.t. $\sup _{\alpha, \beta \in \mathbb{N}_{0}^{d}}\left\|x^{\beta} D^{\alpha} u(x)\right\|_{L^{p}} e^{-\lambda \varphi^{*}\left(\frac{|\alpha|}{\lambda}\right)} e^{-\mu \varphi^{*}\left(\frac{|\beta|}{\mu}\right)} \leq C_{\mu, \lambda} ;$

$(f)^{\prime} \forall \lambda>0 \exists C_{\lambda}>0$ s.t. $\sup _{\alpha, \beta \in \mathbb{N}_{0}^{d}}\left\|x^{\beta} D^{\alpha} u(x)\right\|_{L^{p}} e^{-\lambda \varphi^{*}\left(\frac{|\alpha+\beta|}{\lambda}\right)} \leq C_{\lambda} ;$

$(g)^{\prime} \forall \mu, \lambda>0 \exists C_{\mu, \lambda}>0$ s.t. $\sup _{\alpha \in \mathbb{N}_{0}^{d}}\left\|e^{\mu \omega(x)} D^{\alpha} u(x)\right\|_{L^{p}} e^{-\lambda \varphi^{*}\left(\frac{|\alpha|}{\lambda}\right)} \leq C_{\mu, \lambda} ;$

$(h)^{\prime}$ Given $\psi \in \mathcal{S}_{\omega}\left(\mathbb{R}^{d}\right) \backslash\{0\}, \forall \lambda>0 \exists C_{\lambda}>0$ s.t. $\left\|V_{\psi} u(z) e^{\lambda \omega(z)}\right\|_{L^{p, q}} \leq C_{\lambda}$.

Proof. $(c)^{\prime} \Leftrightarrow u \in \mathcal{S}_{\omega}\left(\mathbb{R}^{d}\right):$

Let us assume $u \in \mathcal{S}\left(\mathbb{R}^{d}\right)$ to satisfy $(c)^{\prime}$ and prove that $u \in \mathcal{S}_{\omega}\left(\mathbb{R}^{d}\right)$. To this aim we shall prove that $u$ satisfies condition $(h)$ of Theorem 2.4 , for some fixed $\psi \in \mathcal{S}_{\omega}\left(\mathbb{R}^{d}\right) \backslash\{0\}$. We fix $\sigma \geq(d+1) / b p^{\prime}$, where $b$ is the constant in condition $(\gamma)$ of Definition 2.1 and $p^{\prime}$ is the conjugate exponent of $p$. Let us first compute

$$
\begin{aligned}
\left|e^{\lambda \omega(x)} V_{\psi} u(x, \xi)\right| & =\left|e^{\lambda \omega(x)} \int_{\mathbb{R}^{d}} u(y) \overline{\psi(y-x)} e^{-i\langle y, \xi\rangle} d y\right| \\
& \leq e^{\lambda L} \int_{\mathbb{R}^{d}}|u(y)| e^{\lambda L \omega(y)}|\psi(y-x)| e^{\lambda L \omega(y-x)+\sigma \omega(y-x)} e^{-\sigma \omega(y-x)} d y \\
& \leq e^{\lambda L}\left\|e^{(\lambda L+\sigma) \omega(t)} \psi(t)\right\|_{L^{\infty}} \cdot\left\|e^{\lambda L \omega(y)} u(y)\right\|_{L^{p}} \cdot\left\|e^{-\sigma \omega(t)}\right\|_{L^{p^{\prime}}} \leq C_{\lambda}
\end{aligned}
$$


since $\psi \in \mathcal{S}_{\omega}\left(\mathbb{R}^{d}\right)$ and because of $(c)^{\prime}(i),(2.1)$ and (2.3).

On the other hand (see [18, formula (3.10)]),

$$
V_{\psi} u(x, \xi)=e^{-i\langle x, \xi\rangle} V_{\hat{\psi}} \hat{u}(\xi,-x),
$$

so that, as in (2.6) with $q$ instead of $p$, we obtain that also

$$
\left|e^{\lambda \omega(\xi)} V_{\psi} u(x, \xi)\right|=\left|e^{\lambda \omega(\xi)} V_{\hat{\psi}} \hat{u}(\xi,-x)\right| \leq C_{\lambda}^{\prime}
$$

for some $C_{\lambda}^{\prime}>0$.

Then, from (2.6), (2.7) and (2.1):

$$
\begin{aligned}
\left|V_{\psi} u(x, \xi)\right| & =\sqrt{\left|V_{\psi} u(x, \xi)\right|^{2}} \leq \sqrt{C_{\lambda} e^{-\lambda \omega(x)} C_{\lambda}^{\prime} e^{-\lambda \omega(\xi)}} \\
& \leq C_{\lambda}^{\prime \prime} e^{-\frac{\lambda}{2}(\omega(x)+\omega(\xi))} \leq C_{\lambda}^{\prime \prime} e^{\frac{\lambda}{2}} e^{-\frac{\lambda}{2 L} \omega(x, \xi)}
\end{aligned}
$$

for some $C_{\lambda}^{\prime \prime}>0$, i.e. condition $(h)$ of Theorem 2.4 is satisfied and $u \in \mathcal{S}_{\omega}\left(\mathbb{R}^{d}\right)$.

Conversely, if $u \in \mathcal{S}_{\omega}\left(\mathbb{R}^{d}\right)$ then condition (c) of Theorem 2.4 is satisfied and hence from (2.3)

$$
\left\|e^{\lambda \omega(x)} u(x)\right\|_{L^{p}} \leq\left\|e^{(\lambda+\sigma) \omega(x)} u(x)\right\|_{L^{\infty}} \cdot\left\|e^{-\sigma \omega(x)}\right\|_{L^{p}} \leq C_{\lambda}
$$

for $\sigma \geq(d+1) / b p$, and analogously $\left\|e^{\lambda \omega(\xi)} \hat{u}(\xi)\right\|_{L^{q}} \leq C_{\lambda}$ for some $C_{\lambda}>0$.

$(a)^{\prime} \Leftrightarrow u \in \mathcal{S}_{\omega}\left(\mathbb{R}^{d}\right):$

If $u$ satisfies $(a)^{\prime}$, then it satisfies $(c)^{\prime}$, so from the previous point $u \in \mathcal{S}_{\omega}\left(\mathbb{R}^{d}\right)$. On the other hand, if $u \in \mathcal{S}_{\omega}\left(\mathbb{R}^{d}\right)$, from $(a)$ of Theorem 2.4 we have

$$
\left\|e^{\lambda \omega(x)} D^{\alpha} u(x)\right\|_{L^{p}} \leq\left\|e^{(\lambda+\sigma) \omega(x)} D^{\alpha} u(x)\right\|_{L^{\infty}}\left\|e^{-\sigma \omega(x)}\right\|_{L^{p}} \leq C_{\alpha, \lambda}^{\prime},
$$

for $\sigma \geq(d+1) / b p$, so $(a)^{\prime}(i)$ is satisfied; the proof of $(a)^{\prime}(i i)$ is similar.

$(b)^{\prime} \Leftrightarrow u \in \mathcal{S}_{\omega}\left(\mathbb{R}^{d}\right):$

It is enough to prove that $(b)^{\prime} \Leftrightarrow(c)^{\prime}$. Since $(b)^{\prime} \Rightarrow(c)^{\prime}$ is trivial, let us suppose that $u$ satisfies $(c)^{\prime}$; from the condition $(\gamma)$ of Definition 2.1 , for $c=1 / b$ and $C_{\alpha}=e^{-a|\alpha| / b}$, we have

$$
\left|e^{\lambda \omega(x)} x^{\alpha}\right| \leq e^{\lambda \omega(x)+|\alpha| \log |x|} \leq C_{\alpha} e^{(\lambda+c|\alpha|) \omega(x)} .
$$

Hence, we obtain

$$
\left\|e^{\lambda \omega(x)} x^{\alpha} u(x)\right\|_{L^{p}} \leq C_{\alpha}\left\|e^{(\lambda+c|\alpha|) \omega(x)} u(x)\right\|_{L^{p}} \leq C_{\alpha, \lambda}
$$

for some $C_{\alpha, \lambda}>0$, so that $(b)^{\prime}(i)$ is satisfied. Analogously we get $(b)^{\prime}(i i)$.

$$
\underline{(f)^{\prime} \Leftrightarrow u \in \mathcal{S}_{\omega}\left(\mathbb{R}^{d}\right):}
$$

Let $u \in \mathcal{S}\left(\mathbb{R}^{d}\right)$ which satisfies $(f)^{\prime}$. It is enough to see that $\hat{u} \in \mathcal{S}_{\omega}\left(\mathbb{R}^{d}\right)$. For all $\xi \in \mathbb{R}^{d}$, $\alpha, \beta \in \mathbb{N}_{0}^{d}$ :

$$
\begin{aligned}
\left|\xi^{\beta} D^{\alpha} \hat{u}(\xi)\right| & =\left|\mathcal{F}\left(D_{x}^{\beta}\left(x^{\alpha} u(x)\right)\right)(\xi)\right| \leq\left\|D^{\beta} x^{\alpha} u\right\|_{L^{1}} \\
& \leq\left\|\left(1+|x|^{2}\right)^{-n}\right\|_{L^{p^{\prime}}} \cdot\left\|\left(1+|x|^{2}\right)^{n} D^{\beta}\left(x^{\alpha} u(x)\right)\right\|_{L^{p}} \\
& \leq C_{n} \sum_{\substack{\gamma \leq \beta \\
\gamma \leq \alpha}}\left(\begin{array}{l}
\beta \\
\gamma
\end{array}\right) \frac{\alpha !}{(\alpha-\gamma) !}\left\|\left(1+|x|^{2}\right)^{n} x^{\alpha-\gamma} D^{\beta-\gamma} u(x)\right\|_{L^{p}}
\end{aligned}
$$


for some $C_{n}>0$ if we choose $n>d /\left(2 p^{\prime}\right)$. Therefore, by $(f)^{\prime}$, it is easy to see (Lemma 4.6) that for every $\lambda>0$ there exists $C_{\lambda}>0$ such that for each $\xi \in \mathbb{R}^{d}$,

$$
\left|\xi^{\beta} D^{\alpha} \hat{u}(\xi)\right| \leq C_{\lambda} e^{\lambda \varphi^{*}\left(\frac{|\alpha+\beta|}{\lambda}\right)} .
$$

In the other direction, if $u \in \mathcal{S}_{\omega}\left(\mathbb{R}^{d}\right)$, we have, by Lemma 4.6,

$$
\begin{aligned}
\left\|x^{\beta} D^{\alpha} u(x)\right\|_{L^{p}} & \leq\left\|\left(1+|x|^{2}\right)^{-n}\right\|_{L^{p}} \cdot\left\|\left(1+|x|^{2}\right)^{n} x^{\beta} D^{\alpha} u(x)\right\|_{L^{\infty}} \\
& \leq C_{n} C_{2 \lambda}^{\prime} e^{2 \lambda \varphi^{*}\left(\frac{|\alpha+\beta|+2 n}{2 \lambda}\right)} \leq C_{n} C_{2 \lambda}^{\prime \prime} e^{\lambda \varphi^{*}\left(\frac{|\alpha+\beta|}{\lambda}\right)} e^{\lambda \varphi^{*}\left(\frac{2 n}{\lambda}\right)} \\
& \leq \widetilde{C}_{\lambda} e^{\lambda \varphi^{*}\left(\frac{|\alpha+\beta|}{\lambda}\right)}
\end{aligned}
$$

for some $C_{n}, C_{2 \lambda}^{\prime}, C_{2 \lambda}^{\prime \prime}, \widetilde{C}_{\lambda}>0$ if we choose $n>d /(2 p)$.

$(e)^{\prime} \Leftrightarrow u \in \mathcal{S}_{\omega}\left(\mathbb{R}^{d}\right):$

From the convexity of $\varphi^{*}$ we get that $(e)^{\prime} \Leftrightarrow(f)^{\prime}$, cf. Lemma 4.6(ii) and (ix).

$(g)^{\prime} \Leftrightarrow u \in \mathcal{S}_{\omega}\left(\mathbb{R}^{d}\right):$

We assume $(g)^{\prime}$ is satisfied and we prove $(e)^{\prime}$. By Lemma $4.6(\mathrm{v})$, for all $\alpha, \beta \in \mathbb{N}_{0}^{d}, \lambda, \mu>0$ :

$$
\begin{aligned}
\left\|x^{\beta} D^{\alpha} u(x)\right\|_{L^{p}} & \leq C_{\mu}\left\|e^{\mu \omega(x)} e^{\mu \varphi^{*}\left(\frac{|\beta|}{\mu}\right)} D^{\alpha} u(x)\right\|_{L^{p}} \\
& \leq C_{\mu} e^{\mu \varphi^{*}\left(\frac{|\beta|}{\mu}\right)}\left\|e^{\mu \omega(x)} D^{\alpha} u(x)\right\|_{L^{p}} \\
& \leq C_{\mu, \lambda} e^{\mu \varphi^{*}\left(\frac{|\beta|}{\mu}\right)} e^{\lambda \varphi^{*}\left(\frac{|\alpha|}{\lambda}\right)}
\end{aligned}
$$

for some $C_{\mu}, C_{\mu, \lambda}>0$.

Let us now assume $u \in \mathcal{S}_{\omega}\left(\mathbb{R}^{d}\right)$. Then condition $(g)$ of Theorem 2.4 is satisfied, and hence for $\sigma \geq(d+1) / b p$ and for every $\alpha \in \mathbb{N}_{0}^{d}$ and $\mu>0$ :

$$
\begin{aligned}
\left\|e^{\mu \omega(x)} D^{\alpha} u(x)\right\|_{L^{p}} & =\left\|e^{(\mu+\sigma) \omega(x)} D^{\alpha} u(x) e^{-\sigma \omega(x)}\right\|_{L^{p}} \\
& \leq\left\|e^{(\mu+\sigma) \omega(x)} D^{\alpha} u(x)\right\|_{L^{\infty}} \cdot\left\|e^{-\sigma \omega(x)}\right\|_{L^{p}} \\
& \leq C_{\mu, \lambda} e^{\lambda \varphi^{*}\left(\frac{|\alpha|}{\lambda}\right)}
\end{aligned}
$$

for some $C_{\mu, \lambda}>0$ by $(g)$ and $(2.3)$.

$$
\underline{(d)^{\prime} \Leftrightarrow u \in \mathcal{S}_{\omega}\left(\mathbb{R}^{d}\right)}:
$$

Let $u \in \mathcal{S}_{\omega}\left(\mathbb{R}^{d}\right)$; then $u$ satisfies $(e)^{\prime}$ for any $p$ (or $\left.q\right)$ in $[1,+\infty]$. Then $(d)^{\prime}$ is trivially satisfied for any $1 \leq p, q \leq+\infty$.

In the opposite direction, we have that, using $(d)^{\prime}(i)$ it is not difficult to see that (Lemma 4.6)

$$
\left|\xi^{\beta} D^{\alpha} \hat{u}(\xi)\right| \leq C_{\alpha, \lambda} e^{\lambda \varphi^{*}\left(\frac{|\beta|}{\lambda}\right)}, \quad \xi \in \mathbb{R}^{d} .
$$

So $\hat{u}$ satisfies $(d)(i i)$ of Theorem 2.4. In the same way, the fact that $u$ satisfies $(d)^{\prime}(i i)$ implies that $\hat{u}$ satisfies $(d)(i)$ of Theorem 2.4. Then $\hat{u} \in \mathcal{S}_{\omega}\left(\mathbb{R}^{d}\right)$.

$(h)^{\prime} \Leftrightarrow u \in \mathcal{S}_{\omega}\left(\mathbb{R}^{d}\right):$

If $\overline{u \in \mathcal{S}_{\omega}\left(\mathbb{R}^{d}\right) \text { then } u}$ satisfies $(h)$ of Theorem 2.4, and so

$$
\left\|V_{\psi} u(z) e^{\lambda \omega(z)}\right\|_{L^{p, q}} \leq\left\|V_{\psi} u(z) e^{(\lambda+\sigma) \omega(z)}\right\|_{L^{\infty}}\left\|e^{-\sigma \omega(z)}\right\|_{L^{p, q}} \leq C_{\lambda}
$$

for $\sigma$ sufficiently large, from (2.3). 
In the opposite direction, we prove that $(h)^{\prime} \Rightarrow(e)$ of Theorem 2.4. From the proof of [12, Proposition 2.10], under condition (2.1) instead of subadditivity, we have

$$
e^{-\lambda \varphi^{*}\left(\frac{|\alpha|}{\lambda}\right)} e^{-\mu \varphi^{*}\left(\frac{|\beta|}{\mu}\right)}\left|y^{\beta} D^{\alpha} u(y)\right| \leq C_{\lambda, \mu} \int_{\mathbb{R}^{2 d}}\left|V_{\psi} u(z)\right| e^{(\mu L+3 L \lambda+\sigma) \omega(z)} e^{-\sigma \omega(z)} d z
$$

for every $\sigma>0$; using Hölder's inequality for $L^{p, q}$ spaces we get

$$
\begin{aligned}
& e^{-\lambda \varphi^{*}\left(\frac{|\alpha|}{\lambda}\right)} e^{-\mu \varphi^{*}\left(\frac{|\beta|}{\mu}\right)}\left|y^{\beta} D^{\alpha} u(y)\right| \\
& \quad \leq C_{\lambda, \mu}\left\|V_{\psi} u(z) e^{(\mu L+3 L \lambda+\sigma) \omega(z)}\right\|_{L^{p, q}}\left\|e^{-\sigma \omega(z)}\right\|_{L^{p^{\prime}, q^{\prime}}} \leq C_{\lambda, \mu}^{\prime},
\end{aligned}
$$

if we choose $\sigma$ sufficiently large, cf. (2.3).

We observe that Theorems 2.4 and 2.5 provide equivalent systems of seminorms for the space $\mathcal{S}_{\omega}\left(\mathbb{R}^{d}\right)$.

\section{Real Paley-Wiener theorems for $\omega$-ultradifferentiable functions}

Now, we prove different "real Paley-Wiener theorems" in the spirit of $[2,4,5]$ in spaces of $\omega$-ultradifferentiable functions. Moreover, we analyze the behavior of time-frequency representations (Gabor and Wigner) of $\omega$-ultradifferentiable functions which have Fourier transform with compact support.

We shall use in the following the notation $\langle f, g\rangle$ for the inner product in $L^{2}$ when $f, g \in L^{2}$, or (more generally) for the duality, that we consider as conjugate linear application of $f$ to $g$. Unless stated otherwise, $\omega$ is a non-quasianalytic weight as in Definition 2.1, $\varphi$ is as in $(\delta)$ in Definition 2.1, and $\varphi^{*}$ is as in (2.4).

Here, we consider, for $R>0$ the space

$$
\mathrm{PW}_{R}^{\omega}\left(\mathbb{R}^{d}\right):=\left\{f \in C^{\infty}\left(\mathbb{R}^{d}\right): \forall \lambda>0, \sup _{\alpha \in \mathbb{N}_{0}^{d}} \sup _{x \in \mathbb{R}^{d}} R^{-|\alpha|} e^{\lambda \omega\left(\frac{x}{|\alpha|+1}\right)}\left|D^{\alpha} f(x)\right|<+\infty\right\}
$$

Lemma 3.1. $\mathrm{PW}_{R}^{\omega}\left(\mathbb{R}^{d}\right) \subseteq \mathcal{S}_{\omega}\left(\mathbb{R}^{d}\right)$.

Proof. Let $f \in \mathrm{PW}_{R}^{\omega}\left(\mathbb{R}^{d}\right)$ and let us first prove that $f \in \mathcal{S}\left(\mathbb{R}^{d}\right)$. Indeed, there exists a constant $C>0$ such that for every $\alpha, \beta \in \mathbb{N}_{0}^{d}$ there exists $C_{\alpha, \beta}>0$ such that

$$
\begin{aligned}
\left|x^{\beta} D^{\alpha} f(x)\right| & \leq C R^{|\alpha|}\left|x^{\beta}\right| e^{-\omega\left(\frac{x}{|\alpha|+1}\right)} \\
& \leq C R^{|\alpha|}|x|^{|\beta|} e^{-\frac{1}{D_{|\alpha|}} \omega(x)+1} \\
& \leq C_{\alpha} R^{|\alpha|} e^{\frac{1}{D_{|\alpha|}} \varphi^{*}\left(|\beta| D_{|\alpha|}\right)}=: C_{\alpha, \beta},
\end{aligned}
$$

by $(2.2)$ and Lemma $4.6(\mathrm{v})$. Now, we prove conditions $(c)(i)$ and $(c)(i i)$ of Theorem 2.4. Condition $(c)(i)$ trivially follows from the definition of $\mathrm{PW}_{R}^{\omega}\left(\mathbb{R}^{d}\right)$ with $\alpha=0$. Let us prove 
condition $(c)(i i)$. For $|\xi| \geq 1$ and $N \in \mathbb{N}_{0}$ we have:

$$
\begin{aligned}
|\hat{f}(\xi)| & =\left|\int_{\mathbb{R}^{d}} f(x) e^{-i\langle x, \xi\rangle} d x\right| \\
& \leq \frac{1}{|\xi|^{2 N}}\left|\int_{\mathbb{R}^{d}} f(x) \Delta_{x}^{N} e^{-i\langle x, \xi\rangle} d x\right| \\
& \leq \frac{1}{|\xi|^{2 N}} \int_{\mathbb{R}^{d}}\left|\Delta_{x}^{N} f(x)\right| d x \\
& \leq \frac{1}{|\xi|^{2 N}} \sum_{|\nu|=N} \frac{N !}{\nu !} \int_{\mathbb{R}^{d}}\left|D_{x}^{2 \nu} f(x)\right| d x
\end{aligned}
$$

where $\nu \in \mathbb{N}^{d}$ and $D_{x}^{2 \nu}=D_{x_{1}}^{2 \nu_{1}} \cdots D_{x_{d}}^{2 \nu_{d}}$. Since $f \in \mathrm{PW}_{R}^{\omega}\left(\mathbb{R}^{d}\right)$ we thus have, for $|\xi| \geq 1$ and $\lambda \geq(d+1) / b$ :

$$
\begin{aligned}
|\hat{f}(\xi)| & \leq \frac{d^{N}}{|\xi|^{2 N}} C_{\lambda} R^{2 N} \int_{\mathbb{R}^{d}} e^{-\lambda \omega\left(\frac{x}{2 N+1}\right)} d x \\
& =\frac{d^{N}}{|\xi|^{2 N}} C_{\lambda} R^{2 N}(2 N+1)^{d} \int_{\mathbb{R}^{d}} e^{-\lambda \omega(y)} d y \\
& =C_{\lambda}^{\prime} \frac{d^{N} R^{2 N}(2 N+1)^{d}}{|\xi|^{2 N}} \\
& \leq C_{\lambda}^{\prime} \frac{\left(2^{d} R \sqrt{d}\right)^{2 N}}{|\xi|^{2 N}} \\
& \leq C_{\lambda^{\prime}}|\xi|^{-2 N} e^{\lambda^{\prime} \varphi^{*}\left(\frac{2 N}{\lambda^{\prime}}\right)}
\end{aligned}
$$

by Lemma 4.6(viii), for some $C_{\lambda}, C_{\lambda}^{\prime}, C_{\lambda^{\prime}}>0$. Taking the infimum over $N \in \mathbb{N}_{0}$ and applying Lemma 4.6(vi) we have that, for all $\mu>0$ there exists $C_{\mu}>0$ such that for all $|\xi| \geq 1$ :

$$
|\hat{f}(\xi)| \leq C_{\mu} e^{-\mu \omega(\xi)} .
$$

Since the above inequality is trivial for $|\xi| \leq 1$, we finally have (c)(ii) and hence $f \in \mathcal{S}_{\omega}\left(\mathbb{R}^{d}\right)$.

In the following result, we denote by

$$
Q_{R}:=\left\{\xi \in \mathbb{R}^{d}:|\xi|_{\infty} \leq R\right\},
$$

where $\xi \in \mathbb{R}^{d}$ and $|\xi|_{\infty}$ is its sup norm.

Theorem 3.2. Let $R>0, f \in C^{\infty}\left(\mathbb{R}^{d}\right)$ and $\omega$ a non-quasianalytic weight function. The following conditions are equivalent:

(a) The function $f \in \mathrm{PW}_{R}^{\omega}\left(\mathbb{R}^{d}\right)$,

(b) The function $f \in \mathcal{S}_{\omega}\left(\mathbb{R}^{d}\right)$ and its Fourier transform $\hat{f}$ is compactly supported with $\operatorname{supp} \hat{f} \subseteq Q_{R}$. 
Proof. (a) $\Rightarrow(\mathrm{b})$. Let $f \in \mathrm{PW}_{R}^{\omega}\left(\mathbb{R}^{d}\right)$. We integrate by parts,

$$
\begin{aligned}
|\hat{f}(\xi)| & =\left|\frac{1}{\xi_{1}^{2 N}+\cdots+\xi_{d}^{2 N}} \int_{\mathbb{R}^{d}} f(x)\left(D_{x_{1}}^{2 N}+\cdots+D_{x_{d}}^{2 N}\right) e^{-i\langle x, \xi\rangle} d x\right| \\
& \leq \frac{1}{\xi_{1}^{2 N}+\cdots+\xi_{d}^{2 N}} \sum_{j=1}^{d} \int_{\mathbb{R}^{d}}\left|D_{x_{j}}^{2 N} f(x)\right| d x .
\end{aligned}
$$

By hypothesis, we have that for every $\lambda>0$ there exists $C_{\lambda}$ such that

$$
\begin{aligned}
|\hat{f}(\xi)| & \leq C_{\lambda} \frac{1}{\xi_{1}^{2 N}+\cdots+\xi_{d}^{2 N}} \sum_{j=1}^{d} R^{2 N} \int_{\mathbb{R}^{d}} e^{-\lambda \omega\left(\frac{x}{2 N+1}\right)} d x \\
& \leq C_{\lambda} \frac{1}{\xi_{1}^{2 N}+\cdots+\xi_{d}^{2 N}} d R^{2 N}(2 N+1)^{d} \int_{\mathbb{R}^{d}} e^{-\lambda \omega(y)} d y=D_{\lambda} \frac{d R^{2 N}(2 N+1)^{d}}{\xi_{1}^{2 N}+\cdots+\xi_{d}^{2 N}},
\end{aligned}
$$

for a constant $D_{\lambda}$ independent of $N$ and $\lambda \geq(d+1) / b$. Now, we observe that for any $\xi \in \mathbb{R}^{d}$ such that $|\xi|_{\infty}>R$ we have $\sqrt[2 N]{\xi_{1}^{2 N}+\cdots+\xi_{d}^{2 N}}>R$, and so supp $\hat{f} \subseteq Q_{R}$.

(b) $\Rightarrow$ (a) Suppose that $\hat{f} \subset \mathcal{S}_{\omega}\left(\mathbb{R}^{d}\right)$ is compactly supported with supp $\hat{f} \subseteq Q_{R}$. By Fourier inversion formula in $\mathcal{S}\left(\mathbb{R}^{d}\right)$, for $x \neq 0$ and $N \in \mathbb{N}_{0}$ :

$$
\begin{aligned}
\left|D^{\alpha} f(x)\right| & =\frac{1}{(2 \pi)^{d}}\left|\int_{\mathbb{R}^{d}} \mathcal{F}\left(D^{\alpha} f\right)(\xi) e^{i\langle x, \xi\rangle} d \xi\right| \\
\leq & \left|\int_{\mathbb{R}^{d}} \xi^{\alpha} \hat{f}(\xi) \frac{1}{|x|^{2 N}} \Delta_{\xi}^{N} e^{i\langle x, \xi\rangle} d \xi\right| \\
\leq & \frac{1}{|x|^{2 N}} \int_{\mathbb{R}^{d}}\left|\Delta_{\xi}^{N} \xi^{\alpha} \hat{f}(\xi)\right| d \xi \\
\leq & \frac{1}{|x|^{2 N}} \int_{\mathbb{R}^{d}} \sum_{|\nu|=N} \frac{N !}{\nu !}\left|D_{\xi_{1}}^{2 \nu_{1}} \cdots D_{\xi_{d}}^{2 \nu_{d}}\left(\xi_{1}^{\alpha_{1}} \cdots \xi_{d}^{\alpha_{d}} \hat{f}(\xi)\right)\right| d \xi \\
\leq & \frac{1}{|x|^{2 N}} \sum_{|\nu|=N} \frac{N !}{\nu !} \sum_{h_{1}=0}^{\min \left\{2 \nu_{1}, \alpha_{1}\right\}}\left(\begin{array}{c}
2 \nu_{1} \\
h_{1}
\end{array}\right) \ldots \sum_{h_{d}=0}^{\min \left\{2 \nu_{d}, \alpha_{d}\right\}}\left(\begin{array}{c}
2 \nu_{d} \\
h_{d}
\end{array}\right) \\
& \frac{\alpha_{1} !}{\left(\alpha_{1}-h_{1}\right) !} \cdots \frac{\alpha_{d} !}{\left(\alpha_{d}-h_{d}\right) !} \int_{|\xi|_{\infty} \leq R}\left|\xi_{1}^{\alpha_{1}-h_{1}} \cdots \xi_{d}^{\alpha_{d}-h_{d}}\right| \cdot\left|D_{\xi}^{2 \nu-h} \hat{f}(\xi)\right| d \xi
\end{aligned}
$$


where we denoted $D_{\xi}^{2 \nu-h}=D_{\xi_{1}}^{2 \nu_{1}-h_{1}} \cdots D_{\xi_{d}}^{2 \nu_{d}-h_{d}}$. Since $\hat{f} \in \mathcal{S}_{\omega}\left(\mathbb{R}^{d}\right)$, there exists $C_{\mu, \lambda}>0$ such that, applying Theorem $2.4(\mathrm{~g})$ in $(3.5)$, for $|x| \geq 1$ and $N \in \mathbb{N}_{0}$ :

$$
\begin{aligned}
\left|D^{\alpha} f(x)\right| \leq & \frac{1}{|x|^{2 N}} \sum_{|\nu|=N} \frac{N !}{\nu !} \sum_{h_{1}=0}^{\min \left\{2 \nu_{1}, \alpha_{1}\right\}}\left(\begin{array}{c}
2 \nu_{1} \\
h_{1}
\end{array}\right) \ldots \sum_{h_{d}=0}^{\min \left\{2 \nu_{d}, \alpha_{d}\right\}}\left(\begin{array}{c}
2 \nu_{d} \\
h_{d}
\end{array}\right) \\
& \cdot \alpha_{1}^{h_{1}} \cdots \alpha_{d}^{h_{d}} \int_{|\xi|_{\infty} \leq R}\left|\xi_{1}\right|^{\alpha_{1}-h_{1}} \ldots\left|\xi_{d}\right|^{\alpha_{d}-h_{d} \mid} D_{\xi}^{2 \nu-h} \hat{f}(\xi) \mid d \xi \\
\leq & \frac{1}{|x|^{2 N}} \sum_{|\nu|=N} \frac{N !}{\nu !} \sum_{h_{1}=0}^{\min \left\{2 \nu_{1}, \alpha_{1}\right\}}\left(\begin{array}{c}
2 \nu_{1} \\
h_{1}
\end{array}\right) \ldots \sum_{h_{d}=0}^{\min \left\{2 \nu_{d}, \alpha_{d}\right\}}\left(\begin{array}{c}
2 \nu_{d} \\
h_{d}
\end{array}\right) \\
& \cdot|\alpha|^{2 N}\left(1+\frac{1}{R}\right)^{2 N} R^{|\alpha|} \int_{\mathbb{R}^{d}} C_{\mu, \lambda} e^{\lambda \varphi^{*}\left(\frac{2 N-|h|}{\lambda}\right)} e^{-\mu \omega(\xi)} d \xi \\
\leq & C_{\lambda} \frac{1}{|x|^{2 N}} d^{N} 2^{2 N}(|\alpha|+1)^{2 N}\left(1+\frac{1}{R}\right)^{2 N} e^{\lambda \varphi^{*}\left(\frac{2 N}{\lambda}\right)} R^{|\alpha|}
\end{aligned}
$$

for some $C_{\lambda}>0$, where we have fixed $\mu \geq(d+1) / b$.

Taking the infimum over $N \in \mathbb{N}_{0}$ and applying Lemma 4.6(vi) we have therefore, for $|x| \geq$ $2 \sqrt{d}(|\alpha|+1)\left(1+\frac{1}{R}\right)$,

$$
\left|D^{\alpha} f(x)\right| \leq C_{\lambda} e^{-\left(\lambda-\frac{2}{b}\right) \omega\left(\frac{x}{2 \sqrt{d}(|\alpha|+1)\left(1+\frac{1}{R}\right)}\right)-\frac{2 a}{b}} R^{|\alpha|}
$$

for $a \in \mathbb{R}, b>0$ as in condition $(\gamma)$ of Definition 2.1.

Let us consider now $|x|<2 \sqrt{d}(|\alpha|+1)\left(1+\frac{1}{R}\right)$. We have

$$
\begin{aligned}
\left|D^{\alpha} f(x)\right| & =\frac{1}{(2 \pi)^{d}}\left|\int_{\mathbb{R}^{d}} \mathcal{F}\left(D^{\alpha} f\right)(\xi) e^{i\langle x, \xi\rangle} d \xi\right| \\
& \leq \int_{Q_{R}}\left|\xi_{1}\right|^{\alpha_{1}} \ldots\left|\xi_{d}\right|^{\alpha_{d}}|\hat{f}(\xi)| d \xi \\
& \leq C R^{|\alpha|},
\end{aligned}
$$

for $C=\|\hat{f}\|_{L^{1}\left(\mathbb{R}^{d}\right)}$. Since $\omega$ is increasing we have that (3.6) is true also for $|x|<2 \sqrt{d}(|\alpha|+$ 1) $\left(1+\frac{1}{R}\right)$, for a constant $C_{\lambda}$ which depends on $\lambda, a, b, R, d$ and $\omega(1)$. By (3.6) and (2.2) we finally have that for every $\lambda^{\prime}>0$ there exists $C_{\lambda^{\prime}}>0$, depending on $\omega, \lambda^{\prime}, d, R, a$ and $b$, such that

$$
\left|D^{\alpha} f(x)\right| \leq C_{\lambda^{\prime}} e^{-\lambda^{\prime} \omega\left(\frac{x}{|\alpha|+1}\right)} R^{|\alpha|}
$$

This proves that $f \in \mathrm{PW}_{R}^{\omega}\left(\mathbb{R}^{d}\right)$.

Let us define, for a function $g$ on $\mathbb{R}^{d}$ :

$$
R_{g}:=\sup \left\{|x|_{\infty}: x \in \operatorname{supp} g\right\} .
$$

The next result treats two different cases: the first one does not need weight functions and it is a natural extension of Theorem 1 of [5] for several variables; in the other case, we assume two different additional conditions on the non-quasianalytic weight function: subadditivity 
(condition (3.9)) or a "mild" condition introduced in [13] that guarantees that the weight does not increase too slowly (condition (3.10)). We shall use in the following the notation $f^{(\alpha)}$ for $D^{\alpha} f$.

Proposition 3.3. Let $1 \leq p \leq+\infty$ and $f \in C^{\infty}\left(\mathbb{R}^{d}\right)$. We have:

(1) If $f^{(\alpha)}(x) \in L^{p}\left(\mathbb{R}^{d}\right)$ for all $\alpha \in \mathbb{N}_{0}^{d}$, we have

$$
\lim _{n \rightarrow+\infty}\left(\max _{|\alpha|=n}\left\|f^{(\alpha)}(x)\right\|_{L^{p}}\right)^{1 / n}=R_{\hat{f}} .
$$

(2) Assume that $e^{\lambda \omega\left(\frac{x}{|\alpha|+1}\right)} f^{(\alpha)}(x) \in L^{p}\left(\mathbb{R}^{d}\right)$ for all $\alpha \in \mathbb{N}_{0}^{d}$ and for some $\lambda>0$, and that the non-quasianalytic weight function $\omega$ satisfies one of the following conditions:

(a) It is subadditive, i.e.,

$$
\omega\left(t_{1}+t_{2}\right) \leq \omega\left(t_{1}\right)+\omega\left(t_{2}\right), \quad t_{1}, t_{2} \geq 0
$$

(b) There is a constant $H>1$ such that

$$
2 \omega(t) \leq \omega(H t)+H, \quad t \geq 0
$$

Then

$$
\lim _{n \rightarrow+\infty}\left(\max _{|\alpha|=n}\left\|e^{\mu \omega\left(\frac{x}{|\alpha|+1}\right)} f^{(\alpha)}(x)\right\|_{L^{p}}\right)^{1 / n}=R_{\hat{f}}, \text { for all } 0 \leq \mu \leq \lambda .
$$

Remark 3.4. We observe that, in general, $R_{\hat{f}} \in\{t \in \mathbb{R} ; t \geq 0\} \cup\{+\infty\}$, so that $\hat{f}$ may not have compact support. Moreover, the limit (3.11) does not depend on $\mu$.

Proof of Proposition 3.3. It suffices to see (2), since (1) can be proved in the same way (it is statement (2) for $\lambda=0$ ). We can assume that $p<\infty$, because the same proof is valid for $p=\infty$ with some small modifications. First, we consider $\phi \in \mathcal{S}_{\omega}\left(\mathbb{R}^{d}\right)$ such that $\hat{\phi}$ has compact support. Then, by Theorem 3.2, we have that $\phi \in \mathrm{PW}_{R_{\hat{\phi}}}^{\omega}\left(\mathbb{R}^{d}\right)$ and hence, for every $1 \leq p<+\infty, \lambda>0$, and $\sigma \geq 2 / b p$ :

$$
\begin{aligned}
\left\|e^{\lambda \omega\left(\frac{x}{|\alpha|+1}\right)} \phi^{(\alpha)}(x)\right\|_{L^{p}}^{1 / n} & =\left\|e^{(\lambda+\sigma) \omega\left(\frac{x}{|\alpha|+1}\right)} \phi^{(\alpha)}(x) e^{-\sigma \omega\left(\frac{x}{|\alpha|+1}\right)}\right\|_{L^{p}}^{1 / n} \\
& \leq\left\|e^{(\lambda+\sigma) \omega\left(\frac{x}{|\alpha|+1}\right)} \phi^{(\alpha)}(x)\right\|_{L^{\infty}}^{1 / n} \cdot\left\|e^{-\sigma \omega\left(\frac{x}{|\alpha|+1}\right)}\right\|_{L^{p}}^{1 / n} \\
& \leq\left(C_{\lambda+\sigma} R_{\hat{\phi}}^{|\alpha|}\right)^{1 / n}(|\alpha|+1)^{\frac{d}{p n}}\left\|e^{-\sigma \omega(x)}\right\|_{L^{p}}^{1 / n}
\end{aligned}
$$

So, if we take the maximum when $|\alpha|=n$ and then the limit when $n$ tends to infinity, we deduce

$$
\limsup _{n \rightarrow+\infty}\left(\max _{|\alpha|=n}\left\|e^{\lambda \omega\left(\frac{x}{|\alpha|+1}\right)} \phi^{(\alpha)}(x)\right\|_{L^{p}}^{1 / n}\right) \leq R_{\hat{\phi}}, \quad p \in[1,+\infty), \lambda>0 .
$$

Now, we consider $f \in C^{\infty}\left(\mathbb{R}^{d}\right)$ such that $e^{\lambda \omega\left(\frac{x}{|\alpha|+1}\right)} f^{(\alpha)}(x) \in L^{p}\left(\mathbb{R}^{d}\right)$ for all $\alpha \in \mathbb{N}_{0}^{d}$. We observe that $f \in \mathcal{S}^{\prime}\left(\mathbb{R}^{d}\right)$ and hence its Fourier transform is well defined. Assume, for the moment, that supp $\hat{f}$ is compact, so that $R_{\hat{f}} \in \mathbb{R}$. 
We observe that if the weight satisfies hypothesis $(2)(a)$, i.e., it is subadditive, we have

$$
\lambda \omega\left(\frac{x}{n+1}\right) \leq \lambda \omega\left(\frac{y}{n+1}\right)+\lambda \omega\left(\frac{x-y}{n+1}\right),
$$

for any $x, y \in \mathbb{R}^{d}, \lambda \geq 0$ and $n \in \mathbb{N}$. On the other hand, it is easy to deduce from hypothesis (2) (b) that for each $k \in \mathbb{N}$,

$$
2^{k} \omega(x) \leq \omega\left(H^{k} x\right)+H\left(2^{k-1}+2^{k-2}+\cdots+1\right), \quad x \in \mathbb{R}^{d},
$$

and hence, $\omega(x) \leq 2^{-k} \omega\left(H^{k} x\right)+H$, for all $x \in \mathbb{R}^{d}$. Now, we take $k \in \mathbb{N}$ so that $L \leq 2^{k}$, where $L \geq 1$ is the constant of condition $(\alpha)$ of Definition 2.1 . Then, we select $n \in \mathbb{N}$ big enough with $H^{k} \leq n+1$ to obtain, from $(2.1)$,

$$
\begin{aligned}
\lambda \omega\left(\frac{x}{n+1}\right) & \leq \lambda L \omega\left(\frac{y}{n+1}\right)+\lambda L \omega\left(\frac{x-y}{n+1}\right)+\lambda L \\
& \leq \lambda L \omega\left(\frac{y}{n+1}\right)+\lambda L 2^{-k} \omega\left(\frac{H^{k}(x-y)}{n+1}\right)+\lambda L+H \\
& \leq \lambda L \omega\left(\frac{y}{n+1}\right)+\lambda \omega(x-y)+\lambda L+H
\end{aligned}
$$

for all $x, y \in \mathbb{R}^{d}$. Hence, under both hypotheses on the weight function $\omega$, we have, by (3.13) or (3.14), for each $x, y \in \mathbb{R}^{d}$ and $n$ big enough,

$$
\lambda \omega\left(\frac{x}{n+1}\right) \leq \lambda L \omega\left(\frac{y}{n+1}\right)+\lambda \omega(x-y)+D_{\lambda},
$$

for some constant $D_{\lambda}$ that depends on $\lambda \geq 0$ and the weight function $\omega$.

Let $\varepsilon>0$ and choose $\phi \in \mathcal{S}_{\omega}\left(\mathbb{R}^{d}\right)$ such that $\hat{\phi} \equiv 1$ in a neighborhood of $\left[-R_{\hat{f}}, R_{\hat{f}}\right]^{d}$ and $\hat{\phi} \equiv 0$ outside $\left[-R_{\hat{f}}-\varepsilon, R_{\hat{f}}+\varepsilon\right]^{d}$. Then $\hat{f}=\hat{f} \cdot \hat{\phi}$ and hence, by the properties of the Fourier transform, $f=f * \phi$. Now, by (3.15), we obtain

$$
\begin{gathered}
\limsup _{n \rightarrow+\infty}\left(\max _{|\alpha|=n}\left\|e^{\lambda \omega\left(\frac{x}{|\alpha|+1}\right)} f^{(\alpha)}(x)\right\|_{L^{p}}^{1 / n}\right)=\limsup _{n \rightarrow+\infty}\left(\max _{|\alpha|=n}\left\|e^{\lambda \omega\left(\frac{x}{n+1}\right)} f * \phi^{(\alpha)}(x)\right\|_{L^{p}}^{1 / n}\right) \\
\quad=\limsup _{n \rightarrow+\infty}\left(\max _{|\alpha|=n}\left\|e^{\lambda \omega\left(\frac{x}{n+1}\right)} \int_{\mathbb{R}^{d}} \phi^{(\alpha)}(y) f(x-y) d y\right\|_{L^{p}}^{1 / n}\right) \\
\leq \limsup _{n \rightarrow+\infty} e^{D_{\lambda} / n}\left(\max _{|\alpha|=n}\left\|\int_{\mathbb{R}^{d}} \phi^{(\alpha)}(y) e^{\lambda L \omega\left(\frac{y}{n+1}\right)} f(x-y) e^{\lambda \omega(x-y)} d y\right\|_{L^{p}}^{1 / n}\right) \\
\leq \limsup _{n \rightarrow+\infty}\left(\max _{|\alpha|=n}\left\|e^{\lambda L \omega\left(\frac{x}{n+1}\right)} \phi^{(\alpha)}(x)\right\|_{L^{1}}^{1 / n}\right)\left\|e^{\lambda \omega(x)} f(x)\right\|_{L^{p}}^{1 / n} \leq R_{\hat{\phi}} \leq R_{\hat{f}}+\varepsilon,
\end{gathered}
$$

since, by assumption, $e^{\lambda \omega(x)} f(x) \in L^{p}\left(\mathbb{R}^{d}\right)$ and, by the construction of $\phi, R_{\hat{\phi}} \leq R_{\hat{f}}+\varepsilon$. Now, as $\varepsilon>0$ is arbitrary, we obtain

$$
\limsup _{n \rightarrow+\infty}\left(\max _{|\alpha|=n}\left\|e^{\lambda \omega\left(\frac{x}{n+1}\right)} f^{(\alpha)}(x)\right\|_{L^{p}}^{1 / n}\right) \leq R_{\hat{f}} .
$$

We remark that when supp $\hat{f}$ is not compact, $R_{\hat{f}}=+\infty$ and, in this case, (3.17) is still valid. 
Take now $0 \neq \xi^{0} \in \operatorname{supp} \hat{f}$, and assume w.l.o.g. that $0<\varepsilon<\left|\xi_{1}^{0}\right|=\left|\xi^{0}\right|_{\infty}$, where $\xi^{0}=$ $\left(\xi_{1}^{0}, \ldots, \xi_{d}^{0}\right) \in \mathbb{R}^{d}$. We take $\psi \in \mathcal{D}_{(\omega)}\left(\mathbb{R}^{d}\right)$ with $\Pi_{1} \operatorname{supp} \psi \subseteq\left[\xi_{1}^{0}-\frac{\varepsilon}{2}, \xi_{1}^{0}+\frac{\varepsilon}{2}\right]$ and $\langle\hat{f}, \psi\rangle \neq 0$, where $\Pi_{1}: \mathbb{R}^{d} \rightarrow \mathbb{R}$ is the projection in the first variable.

Then, for $\xi \in \mathbb{R}^{d}$ with $\xi_{1} \neq 0, \lambda>0$ and $1 \leq p<+\infty$ we have:

$$
\begin{aligned}
& \left(\left|\xi_{1}^{0}\right|-\varepsilon\right)^{n}|\langle\hat{f}(\xi), \psi(\xi)\rangle|=\left(\left|\xi_{1}^{0}\right|-\varepsilon\right)^{n}\left|\left\langle\xi_{1}^{n} \hat{f}(\xi), \xi_{1}^{-n} \psi(\xi)\right\rangle\right| \\
& \quad=\left(\left|\xi_{1}^{0}\right|-\varepsilon\right)^{n}\left|\left\langle\widehat{D_{1}^{n} f}(\xi), \xi_{1}^{-n} \psi(\xi)\right\rangle\right| \\
& \quad=\left(\left|\xi_{1}^{0}\right|-\varepsilon\right)^{n}\left|\left\langle D_{1}^{n} f(x), \mathcal{F}^{-1}\left(\xi_{1}^{-n} \psi(\xi)\right)(x)\right\rangle\right| \\
& \quad \leq\left(\left|\xi_{1}^{0}\right|-\varepsilon\right)^{n}\left\|D_{1}^{n} f\right\|_{L^{p}}\left\|\mathcal{F}^{-1}\left(\xi_{1}^{-n} \psi(\xi)\right)\right\|_{L^{p^{\prime}}} \\
& \quad \leq\left(\left|\xi_{1}^{0}\right|-\varepsilon\right)^{n}\left\|e^{\lambda \omega\left(\frac{x}{n+1}\right)} D_{1}^{n} f(x)\right\|_{L^{p}}\left\|\frac{1}{\left(1+|x|^{2}\right)^{d}} \mathcal{F}^{-1}\left[\left(1-\Delta_{\xi}\right)^{d}\left(\xi_{1}^{-n} \psi(\xi)\right)\right]\right\|_{L^{p^{\prime}}} \\
& \quad \leq\left(\left|\xi_{1}^{0}\right|-\varepsilon\right)^{n}\left\|e^{\lambda \omega\left(\frac{x}{n+1}\right)} D_{1}^{n} f(x)\right\|_{L^{p}}\left\|\frac{1}{\left(1+|x|^{2}\right)^{d}}\right\|_{L^{p^{\prime}}}\left\|\mathcal{F}^{-1}\left[\left(1-\Delta_{\xi}\right)^{d}\left(\xi_{1}^{-n} \psi(\xi)\right)\right]\right\|_{L^{\infty}} .
\end{aligned}
$$

We have

$$
\begin{gathered}
\mathcal{F}^{-1}\left[\left(1-\Delta_{\xi}\right)^{d}\left(\xi_{1}^{-n} \psi(\xi)\right)\right]=\sum_{|\nu|=\nu_{1}+\cdots+\nu_{d+1}=d} \frac{d !}{\nu !} \mathcal{F}^{-1}\left[D_{\xi_{1}}^{2 \nu_{1}} \cdots D_{\xi_{d}}^{2 \nu_{d}}\left(\xi_{1}^{-n} \psi(\xi)\right)\right] \\
=\sum_{|\nu|=d} \frac{d !}{\nu !} \sum_{h=0}^{2 \nu_{1}}\left(\begin{array}{c}
2 \nu_{1} \\
h
\end{array}\right) i^{h} \frac{(n+h-1) !}{(n-1) !} \mathcal{F}^{-1}\left[\xi_{1}^{-n-h} D_{\xi_{1}}^{2 \nu_{1}-h} \cdots D_{\xi_{d}}^{2 \nu_{d}} \psi(\xi)\right] .
\end{gathered}
$$

Therefore, we obtain

$$
\begin{aligned}
& \left\|\mathcal{F}^{-1}\left[\left(1-\Delta_{\xi}\right)^{d}\left(\xi_{1}^{-n} \psi(\xi)\right)\right]\right\|_{L^{\infty}} \\
& \leq 4^{d}(d+1)^{d}(n+2 d)^{2 d} \max _{|\nu|=d, 0 \leq h \leq 2 \nu_{1}}\left\|\xi_{1}^{-n-h} D_{\xi_{1}}^{2 \nu_{1}-h} \cdots D_{\xi_{d}}^{2 \nu_{d}} \psi(\xi)\right\|_{L^{1}} \\
& \leq \frac{1}{\left(\left|\xi_{1}^{0}\right|-\varepsilon / 2\right)^{n}} 4^{d}(d+1)^{d}(n+2 d)^{2 d} \max _{|\nu|=d, 0 \leq h \leq 2 \nu_{1}}\left\|\xi_{1}^{-h} D_{\xi_{1}}^{2 \nu_{1}-h} \cdots D_{\xi_{d}}^{2 \nu_{d}} \psi(\xi)\right\|_{L^{1}}
\end{aligned}
$$

We then obtain

$$
\begin{aligned}
& \left(\left|\xi_{1}^{0}\right|-\varepsilon\right)|\langle\hat{f}, \psi\rangle|^{1 / n} \\
& \leq \frac{\left|\xi_{1}^{0}\right|-\varepsilon}{\left|\xi_{1}^{0}\right|-\varepsilon / 2}\left\|e^{\lambda \omega\left(\frac{x}{n+1}\right)} D_{1}^{n} f(x)\right\|_{L^{p}}^{1 / n}\left\|\frac{1}{\left(1+|x|^{2}\right)^{d}}\right\|_{L^{p^{\prime}}}^{1 / n}(n+2 d)^{2 d / n} C(\psi)^{1 / n},
\end{aligned}
$$

for a constant $C(\psi)$ that depends on $\psi$, the support of $\psi$ and its partial derivatives up to the order $2 d$, and the dimension $d$. Hence, since $\frac{\left|\xi_{1}^{0}\right|-\varepsilon}{\left|\xi_{1}^{0}\right|-\varepsilon / 2} \leq 1$,

$$
\begin{array}{r}
\left|\xi^{0}\right|_{\infty}-\varepsilon \leq \liminf _{n \rightarrow+\infty}\left(\max _{|\alpha|=n}\left\|e^{\lambda \omega\left(\frac{x}{n+1}\right)} f^{(\alpha)}(x)\right\|_{L^{p}}^{1 / n}\right) \\
\leq \limsup _{n \rightarrow+\infty}\left(\max _{|\alpha|=n}\left\|e^{\lambda \omega\left(\frac{x}{n+1}\right)} f^{(\alpha)}(x)\right\|_{L^{p}}^{1 / n}\right) \leq R_{\hat{f}}
\end{array}
$$

by (3.17). 
By the arbitrariness of $\varepsilon>0$ and then of $\xi^{0} \in \operatorname{supp} \hat{f}$ :

$$
\begin{aligned}
R_{\hat{f}} & \leq \liminf _{n \rightarrow+\infty}\left(\max _{|\alpha|=n}\left\|e^{\lambda \omega\left(\frac{x}{n+1}\right)} f^{(\alpha)}(x)\right\|_{L^{p}}^{1 / n}\right) \\
& \leq \limsup _{n \rightarrow+\infty}\left(\max _{|\alpha|=n}\left\|e^{\lambda \omega\left(\frac{x}{n+1}\right)} f^{(\alpha)}(x)\right\|_{L^{p}}^{1 / n}\right) \leq R_{\hat{f}}
\end{aligned}
$$

and, hence, there exists

$$
\lim _{n \rightarrow+\infty}\left(\max _{|\alpha|=n}\left\|e^{\lambda \omega\left(\frac{x}{n+1}\right)} f^{(\alpha)}(x)\right\|_{L^{p}}^{1 / n}\right)=R_{\hat{f}},
$$

for $\lambda>0$ and $1 \leq p<+\infty$.

Remark 3.5. The condition $e^{\lambda \omega\left(\frac{x}{|\alpha|+1}\right)} f^{(\alpha)}(x) \in L^{p}$ for all $\lambda \geq 0$ is equivalent to $e^{\lambda \omega(x)} f^{(\alpha)}(x) \in$ $L^{p}$ for all $\lambda \geq 0$ by (2.2). Therefore, if in Proposition 3.3 we ask that $e^{\lambda \omega(x)} f^{(\alpha)}(x) \in L^{p}\left(\mathbb{R}^{d}\right)$ for all $\alpha \in \mathbb{N}_{0}^{d}$ and all $\lambda \geq 0$, (3.11) is true without the additional assuptions (3.9) or (3.10). Indeed, in (3.16) we can use (2.1) directly.

As we have already mentioned, Proposition 3.3 in the case $\lambda=0$ is [5, Theorem 1] for several variables, cf. [2, Theorem 3] also. On the other hand, we are interested in the case $\lambda>0$ in order to get Paley-Wiener theorems for ultradifferentiable functions; see Theorem 3.17 below. To this aim, first we prove that, under the assumptions of Proposition 3.3, if (3.11) is satisfied for some $R_{\hat{f}} \in \mathbb{R}$ and for all $\lambda>0$, then $u \in \mathcal{S}_{\omega}\left(\mathbb{R}^{d}\right)$. We need some lemmas.

Lemma 3.6. Let $f \in C^{\infty}\left(\mathbb{R}^{d}\right)$ such that $e^{\lambda \omega(x)} f^{(\alpha)}(x) \in L^{p}\left(\mathbb{R}^{d}\right)$ for all $\alpha \in \mathbb{N}_{0}^{d}, \lambda>0$, and some $1 \leq p \leq+\infty$. Then $f \in \mathcal{S}\left(\mathbb{R}^{d}\right)$.

Proof. Since $f \in \mathcal{S}^{\prime}\left(\mathbb{R}^{d}\right)$, we can apply the Fourier transform to $f$. We fix $\alpha, \beta \in \mathbb{N}_{0}^{d}$ and choose $\lambda>0$ big enough such that $x^{\beta-\gamma} e^{-\lambda \omega\left(\frac{x}{|\alpha-\gamma|+1}\right)} \in L^{p^{\prime}}\left(\mathbb{R}^{d}\right)$, for every $\gamma \leq \min \{\alpha, \beta\}$ and for $1 / p+1 / p^{\prime}=1$, and we apply Hölder's inequality to obtain

$$
\begin{aligned}
\left|\xi^{\alpha} D^{\beta} \hat{f}(\xi)\right| & =\left|\mathcal{F}\left(D_{x}^{\alpha}\left(x^{\beta} f(x)\right)\right)(\xi)\right| \leq \int_{\mathbb{R}^{d}}\left|D_{x}^{\alpha}\left(x^{\beta} f(x)\right)\right| d x \\
& \leq \sum_{\substack{\gamma \leq \alpha \\
\gamma \leq \beta}}\left(\begin{array}{l}
\alpha \\
\gamma
\end{array}\right) \frac{\beta !}{(\beta-\gamma) !} \int_{\mathbb{R}^{d}}\left|x^{\beta-\gamma} D_{x}^{\alpha-\gamma} f(x)\right| d x \\
& =\sum_{\substack{\gamma \leq \alpha \\
\gamma \leq \beta}}\left(\begin{array}{l}
\alpha \\
\gamma
\end{array}\right) \frac{\beta !}{(\beta-\gamma) !} \int_{\mathbb{R}^{d}}\left|e^{\lambda \omega\left(\frac{x}{|\alpha-\gamma|+1}\right)} D_{x}^{\alpha-\gamma} f(x)\right| \cdot\left|x^{\beta-\gamma} e^{-\lambda \omega\left(\frac{x}{|\alpha-\gamma|+1}\right)}\right| d x \\
& \leq \sum_{\gamma \leq \alpha}\left(\begin{array}{l}
\alpha \\
\gamma
\end{array}\right) \frac{\beta !}{(\beta-\gamma) !}\left\|e^{\lambda \omega\left(\frac{x}{|\alpha-\gamma|+1}\right)} f^{(\alpha-\gamma)}(x)\right\|_{L^{p}} \cdot\left\|x^{\beta-\gamma} e^{-\lambda \omega\left(\frac{x}{|\alpha-\gamma|+1}\right)}\right\|_{L^{p^{\prime}}} \leq C_{\alpha, \beta, \lambda},
\end{aligned}
$$

which finishes the proof. 
Lemma 3.7. Let $1 \leq p \leq+\infty$ and $f \in C^{\infty}\left(\mathbb{R}^{d}\right)$ with $e^{\lambda \omega(x)} f^{(\alpha)}(x) \in L^{p}\left(\mathbb{R}^{d}\right)$ for all $\alpha \in \mathbb{N}_{0}^{d}$ and for all $\lambda>0$. If $\hat{f}$ has compact support, we have

$$
\sup _{\xi \in \mathbb{R}^{d}} e^{\lambda \omega(\xi)}|\hat{f}(\xi)|<+\infty, \quad \text { for all } \lambda>0 .
$$

Proof. Assume that $\xi=\left(\xi_{1}, \ldots, \xi_{d}\right) \neq 0$, and that $|\xi|_{\infty}=\left|\xi_{1}\right|$. Given $n \in \mathbb{N}_{0}$ and $\lambda \geq \frac{d+1}{b p^{\prime}}$, where $1 / p+1 / p^{\prime}=1$, we can write

$$
\begin{aligned}
|\hat{f}(\xi)| & =\frac{1}{\left|\xi_{1}\right|^{n}}\left|\int_{\mathbb{R}^{d}} f(x) D_{x_{1}}^{n} e^{-i x \xi} d x\right| \\
& \leq \frac{1}{\left|\xi_{1}\right|^{n}} \int_{\mathbb{R}^{d}} e^{\lambda \omega\left(\frac{x}{n+1}\right)}\left|D_{1}^{n} f(x)\right| e^{-\lambda \omega\left(\frac{x}{n+1}\right)} d x \\
& \leq \frac{1}{\left|\xi_{1}\right|^{n}}\left\|e^{\lambda \omega\left(\frac{x}{n+1}\right)} D_{1}^{n} f(x)\right\|_{L^{p}} \cdot\left\|e^{-\lambda \omega\left(\frac{x}{n+1}\right)}\right\|_{L^{p^{\prime}}} \\
& =\frac{C_{\lambda}}{\left|\xi_{1}\right|^{n}}(n+1)^{\frac{d}{p^{\prime}}}\left\|e^{\lambda \omega\left(\frac{x}{n+1}\right)} D_{1}^{n} f(x)\right\|_{L^{p}}
\end{aligned}
$$

for $C_{\lambda}=\left\|e^{-\lambda \omega(x)}\right\|_{L^{p^{\prime}}}<+\infty$ from $(2.3)$.

Since $\hat{f}$ has compact support by assumption, by Proposition 3.3 and Remark 3.5, we have that (3.11) is satisfied with $R_{\hat{f}} \in \mathbb{R}$. Therefore, there exists a constant $D \in \mathbb{R}$, depending only on $f$, such that, for all $n \in \mathbb{N}_{0}$,

$$
\left\|e^{\lambda \omega\left(\frac{x}{n+1}\right)} D_{1}^{n} f(x)\right\|_{L^{p}} \leq D^{n}
$$

and hence, by Lemma 4.6(viii),

$$
|\hat{f}(\xi)| \leq C_{\lambda} \frac{D^{n}}{\left|\xi_{1}\right|^{n}}(n+1)^{\frac{d}{p^{\prime}}} \leq C_{\lambda} \frac{\tilde{D}^{n}}{\left|\xi_{1}\right|^{n}} n ! \leq C_{\lambda}^{\prime}\left|\xi_{1}\right|^{-n} e^{\lambda \varphi^{*}\left(\frac{n}{\lambda}\right)}
$$

for some $\tilde{D}, C_{\lambda}^{\prime}>0$.

Now, by Lemma 4.6(vi), if we assume $\left|\xi_{1}\right| \geq 1$,

$$
|\hat{f}(\xi)| \leq C_{\lambda}^{\prime} e^{-\left(\lambda-\frac{1}{b}\right) \omega\left(\xi_{1}\right)-\frac{a}{b}}
$$

Hence, it is suffices to take $\lambda>1 / b$ big enough to finish the proof.

Lemma 3.8. Let $1 \leq p \leq+\infty$ and $f \in C^{\infty}\left(\mathbb{R}^{d}\right)$ such that $e^{\lambda \omega(x)} f^{(\alpha)}(x) \in L^{p}(\mathbb{R})$ for all $\alpha \in \mathbb{N}_{0}^{d}$ and $\lambda>0$. If $\hat{f}$ has compact support, then $f \in \mathcal{S}_{\omega}\left(\mathbb{R}^{d}\right)$.

Proof. By Lemmas 3.6 and 3.7 we have that $f \in \mathcal{S}\left(\mathbb{R}^{d}\right)$ and, for every $\lambda>0$, there exists $C_{\lambda}>0$ such that $\left\|e^{\lambda \omega(\xi)} \hat{f}(\xi)\right\|_{L^{\infty}} \leq C_{\lambda}$. Moreover $\left\|e^{\lambda \omega(x)} f(x)\right\|_{L^{p}} \leq C_{\lambda}^{\prime}$ for some $C_{\lambda}^{\prime}>0$ by assumption. It follows, from Theorem $2.5(c)^{\prime}$ with $q=\infty$, that $f \in \mathcal{S}_{\omega}\left(\mathbb{R}^{d}\right)$.

3.1. Relation with the Wigner transform. Proposition 3.3 proves that the radius of the support of $\hat{f}$ can be computed with the limit (3.11) for any $\lambda \geq 0$. Now, we give a characterization of the support of $\hat{f}$ in terms of the Wigner transform. First, we introduce the following real Paley-Wiener space defined by means of the Gabor transform: 
Definition 3.9. Let $T, R>0$ and define, for $\psi \in \mathrm{PW}_{T}^{\omega}\left(\mathbb{R}^{d}\right)$,

$\mathrm{PWG}_{R}^{\omega, \psi}\left(\mathbb{R}^{d}\right):=\left\{f \in C^{\infty}\left(\mathbb{R}^{d}\right) \cap \mathcal{S}_{\omega}^{\prime}\left(\mathbb{R}^{d}\right):\right.$ for each $\lambda, \mu>0$,

$$
\left.\sup _{N \in \mathbb{N}_{0}} \sup _{x, \xi \in \mathbb{R}^{d}}(R+T)^{-N} \frac{1}{(N+1)^{d / 2}} e^{\lambda \omega\left(\frac{x}{N+1}\right)+\mu \omega(\xi)}|\xi|_{\infty}^{N}\left|V_{\psi} f(x, \xi)\right|<+\infty\right\} .
$$

Proposition 3.10. Let $\psi \in \mathrm{PW}_{T}^{\omega}\left(\mathbb{R}^{d}\right)$. Then

$$
\mathrm{PW}_{R}^{\omega}\left(\mathbb{R}^{d}\right) \subseteq \mathrm{PWG}_{R}^{\omega, \psi}\left(\mathbb{R}^{d}\right)
$$

Proof. Let $f \in \mathrm{PW}_{R}^{\omega}\left(\mathbb{R}^{d}\right)$. Fix $\xi \in \mathbb{R}^{d} \backslash\{0\}$. Then $|\xi|_{\infty}=\left|\xi_{j}\right|$ for some $1 \leq j \leq d$ and hence

$$
\begin{aligned}
|\xi|_{\infty}^{N}\left|V_{\psi} f(x, \xi)\right| & =\left|\xi_{j}^{N} V_{\psi} f(x, \xi)\right|=\left|\xi_{j}^{N} \int_{\mathbb{R}^{d}} f(y) \overline{\psi(y-x)} e^{-i\langle y, \xi\rangle} d y\right| \\
& =\left|\int_{\mathbb{R}^{d}} f(y) \overline{\psi(y-x)} D_{y_{j}}^{N}\left(e^{-i\langle y, \xi\rangle}\right) d y\right| \\
& =\left|\int_{\mathbb{R}^{d}} D_{y_{j}}^{N}(f(y) \overline{\psi(y-x)}) e^{-i\langle y, \xi\rangle} d y\right| \\
& \leq \sum_{k=0}^{N}\left(\begin{array}{c}
N \\
k
\end{array}\right) \int_{\mathbb{R}^{d}}\left|D_{y_{j}}^{k} f(y)\right| \cdot\left|D_{y_{j}}^{N-k} \psi(y-x)\right| d y
\end{aligned}
$$

Since $f \in \mathrm{PW}_{R}^{\omega}\left(\mathbb{R}^{d}\right)$ and $\psi \in \mathrm{PW}_{T}^{\omega}\left(\mathbb{R}^{d}\right)$, it is not difficult to see that for every $\lambda>0$ there is $C_{\lambda}>0$ such that

$$
|\xi|_{\infty}^{N}\left|V_{\psi} f(x, \xi)\right| \leq C_{\lambda}(R+T)^{N} e^{-\lambda \omega\left(\frac{x}{N+1}\right)}(N+1)^{d},
$$

for all $x, \xi \in \mathbb{R}^{d}$ and $N \in \mathbb{N}_{0}$.

Moreover, since $f, \psi \in \mathcal{S}_{\omega}\left(\mathbb{R}^{d}\right)$ by Lemma 3.1, then $V_{\psi} f \in \mathcal{S}_{\omega}\left(\mathbb{R}^{2 d}\right)$ also $([19$, Thm. 2.7]) and, hence, for all $\mu>0$ there exists $C_{\mu}>0$ such that

$$
\left|V_{\psi} f(x, \xi)\right| \leq C_{\mu} e^{-\mu \omega(\xi)}
$$

since $\omega(x, \xi) \geq \omega(\xi)$.

By Theorem 3.2 we have that $\operatorname{supp} \hat{f} \subseteq Q_{R}$, supp $\hat{\psi} \subseteq Q_{T}$ and hence the projection on $\xi$ of the support of $V_{\psi} f$ satisfies

$$
\Pi_{\xi}\left(\operatorname{supp} V_{\psi} f(x, \xi)\right) \subseteq Q_{R+T}, \quad x \in \mathbb{R}^{d},
$$

as it can be deduced for example from [18, formula (3.8)]. From (3.21) and (3.22) we have that

$$
|\xi|_{\infty}^{N}\left|V_{\psi} f(x, \xi)\right| \leq C_{\mu}(R+T)^{N} e^{-\mu \omega(\xi)}
$$

for all $x, \xi \in \mathbb{R}^{d}, N \in \mathbb{N}_{0}$ and $\mu>0$.

Combining (3.20) and (3.23) we finally have:

$$
\begin{aligned}
|\xi|_{\infty}^{N}\left|V_{\psi} f(x, \xi)\right| & =\sqrt{\left(|\xi|_{\infty}^{N}\left|V_{\psi} f(x, \xi)\right|\right)^{2}} \\
& \leq \sqrt{C_{\lambda}(R+T)^{N} e^{-\lambda \omega\left(\frac{x}{N+1}\right)}(N+1)^{d} C_{\mu}(R+T)^{N} e^{-\mu \omega(\xi)}} \\
& \leq C_{\lambda, \mu}(R+T)^{N}(N+1)^{d / 2} e^{-\frac{\lambda}{2} \omega\left(\frac{x}{N+1}\right)} e^{-\frac{\mu}{2} \omega(\xi)}
\end{aligned}
$$

for some $C_{\lambda, \mu}>0$ and for all $x, \xi \in \mathbb{R}^{d}, N \in \mathbb{N}_{0}, \lambda, \mu>0$. Therefore $f \in \mathrm{PWG}_{R}^{\omega, \psi}\left(\mathbb{R}^{d}\right)$. 
Given the space defined in (2.5), we have the following result:

Proposition 3.11. Let $f, \psi \in \mathcal{S}_{\omega}\left(\mathbb{R}^{d}\right)$ and $p, q \in[1,+\infty]$. Then, for every $\lambda, \mu \geq 0$,

$$
\limsup _{N \rightarrow+\infty}\left\|e^{\lambda \omega\left(\frac{x}{N+1}\right)+\mu \omega(\xi)}|\xi|_{\infty}^{N} V_{\psi} f(x, \xi)\right\|_{L^{p, q}}^{1 / N} \leq R_{\hat{f}}+R_{\hat{\psi}}
$$

Proof. If $\operatorname{supp} \hat{f}$ or $\operatorname{supp} \hat{\psi}$ are not compact, then $R_{\hat{f}}=+\infty$ or, respectively, $R_{\hat{\psi}}=+\infty$, so the inequality (3.24) is trivial. So, we can assume that $\operatorname{supp} \hat{f}$ and $\operatorname{supp} \hat{\psi}$ are compact, and hence $R_{\hat{f}}, R_{\hat{\psi}} \in \mathbb{R}$. By Theorem 3.2 and Proposition 3.10, we have $f \in \mathrm{PW}_{R_{\hat{f}}}^{\omega}\left(\mathbb{R}^{d}\right) \subseteq \mathrm{PWG}_{R_{\hat{f}}}^{\omega, \psi}\left(\mathbb{R}^{d}\right)$ and hence for $\sigma$ and $\tau$ sufficiently large, from (2.3) we obtain

$$
\begin{aligned}
& \limsup _{N \rightarrow+\infty}\left\|e^{\lambda \omega\left(\frac{x}{N+1}\right)+\mu \omega(\xi)}|\xi|_{\infty}^{N} V_{\psi} f(x, \xi)\right\|_{L^{p, q}}^{1 / N} \\
& \leq \limsup _{N \rightarrow+\infty}\left\|e^{(\lambda+\sigma) \omega\left(\frac{x}{N+1}\right)+(\mu+\tau) \omega(\xi)}|\xi|_{\infty}^{N} V_{\psi} f(x, \xi)\right\|_{L^{\infty}}^{1 / N} \cdot\left\|e^{-\sigma \omega\left(\frac{x}{N+1}\right)-\tau \omega(\xi)}\right\|_{L^{p, q}}^{1 / N} \\
& \leq \limsup _{N \rightarrow+\infty} C_{\lambda, \mu}^{\frac{1}{N}}\left(R_{\hat{f}}+R_{\hat{\psi}}\right)(N+1)^{\frac{d}{2 N}+\frac{d}{p N}}\left\|e^{-\sigma \omega(x)-\tau \omega(\xi)}\right\|_{L^{p, q}}^{1 / N}=R_{\hat{f}}+R_{\hat{\psi}},
\end{aligned}
$$

for some $C_{\lambda, \mu}>0$, if $p<+\infty$. If $p=+\infty$ the proof is similar.

We introduce now the following notation for the translation and modulation operators; for $x, \xi, x^{0}, \xi^{0} \in \mathbb{R}^{d}$ we denote

$$
T_{x^{0}} f(x)=f\left(x-x^{0}\right), \quad M_{\xi^{0}} f(\xi)=e^{i\left\langle\xi^{0}, \xi\right\rangle} f(\xi) .
$$

Example 3.12. The inequality (3.24) is strict, in general. Let us consider, for instance, $f \in \mathcal{S}_{\omega}(\mathbb{R})$ with supp $\hat{f} \subseteq\left[R_{\hat{f}}-\mu, R_{\hat{f}}\right]$ for some $0<\mu<R_{\hat{f}}<+\infty$. Then

$$
\left\||\xi|_{\infty}^{N} V_{f} f(x, \xi)\right\|_{L^{p, q}}^{1 / N} \leq \mu\left\|V_{f} f(x, \xi)\right\|_{L^{p, q}}^{1 / N}
$$

since

$$
\Pi_{\xi} \operatorname{supp} V_{f} f(x, \xi)=\cup_{x \in \mathbb{R}} \operatorname{supp}\left(\hat{f} * M_{-x} \overline{\tilde{\hat{f}}}\right)(\xi) \subseteq\left[R_{\hat{f}}-\mu, R_{\hat{f}}\right]+\left[-R_{\hat{f}},-R_{\hat{f}}+\mu\right]=[-\mu, \mu],
$$

where $\tilde{f}(x)=f(-x)$, by $\left[18\right.$, Lemma 3.1.1]. Since $\left\|V_{f} f(x, \xi)\right\|_{L^{p, q}}$ does not depend on $N$, letting $N \rightarrow+\infty$ in $(3.25)$ we get that

$$
\limsup _{N \rightarrow+\infty}\left\||\xi|_{\infty}^{N} V_{f} f(x, \xi)\right\|_{L^{p, q}}^{1 / N} \leq \mu<R_{\hat{f}}<2 R_{\hat{f}}
$$

On the other hand, for the right choice of the window function we get the equality in (3.24), as the next result shows. This fact becomes crucial for the analysis of real Paley-Wiener theorems in terms of the Wigner transform. In the next result the number $R_{\hat{f}}$ could be $+\infty$.

Proposition 3.13. Let $f \in \mathcal{S}_{\omega}\left(\mathbb{R}^{d}\right)$ and $p, q \in[1,+\infty]$. Then, for all $\lambda, \mu \geq 0$, we have

$$
\lim _{N \rightarrow+\infty}\left\|e^{\lambda \omega\left(\frac{x}{N+1}\right)+\mu \omega(\xi)}|\xi|_{\infty}^{N} V_{\tilde{f}} f(x, \xi)\right\|_{L^{p, q}}^{1 / N}=2 R_{\hat{f}}
$$


Proof. By Proposition 3.11 we have

$$
\limsup _{N \rightarrow+\infty}\left\|e^{\lambda \omega\left(\frac{x}{N+1}\right)+\mu \omega(\xi)}|\xi|_{\infty}^{N} V_{\tilde{f}} f(x, \xi)\right\|_{L^{p, q}}^{1 / N} \leq 2 R_{\hat{f}}
$$

since $\hat{\tilde{f}}(\xi)=\hat{f}(-\xi)$ and hence $R_{\hat{\tilde{f}}}=R_{\hat{f}}$.

Now, we fix $\xi^{0} \in \operatorname{supp} \hat{f}$ and $0<\varepsilon<2\left|\xi^{0}\right|_{\infty}$, choose $\phi_{\varepsilon}, \psi_{\varepsilon} \in \mathcal{S}_{\omega}\left(\mathbb{R}^{d}\right)$ with $\operatorname{supp} \hat{\phi}_{\varepsilon}, \hat{\psi}_{\varepsilon} \subseteq Q_{\varepsilon / 4}$ and

$$
\left\langle\hat{f}, T_{\xi^{0}} \hat{\psi}_{\varepsilon}\right\rangle \neq 0, \quad\left\langle\hat{f}, T_{\xi^{0}} \hat{\tilde{\phi}}_{\varepsilon}\right\rangle \neq 0
$$

Note that, by [18, formula (3.10)],

$$
\begin{aligned}
\Pi_{\xi} \operatorname{supp}\left(V_{\phi_{\varepsilon}}\left(M_{2 \xi^{0}} \psi_{\varepsilon}\right)(x, \xi)\right) & =\Pi_{\xi} \operatorname{supp}\left(e^{-i\langle x, \xi\rangle} V_{\hat{\phi}_{\varepsilon}}\left(\widehat{M_{2 \xi^{0}} \psi_{\varepsilon}}\right)(\xi,-x)\right) \\
& =\Pi_{\xi} \operatorname{supp}\left(V_{\hat{\phi}_{\varepsilon}}\left(T_{2 \xi^{0}} \hat{\psi}_{\varepsilon}\right)(\xi,-x)\right) \\
& =\Pi_{\xi} \operatorname{supp}\left(\left(T_{2 \xi^{0}} \hat{\psi}_{\varepsilon} * M_{-x} \widehat{\bar{\phi}}_{\varepsilon}\right)(\xi)\right) \\
& \subseteq Q_{\frac{\varepsilon}{2}}\left(2 \xi^{0}\right):=\left\{\xi \in \mathbb{R}^{d}:\left|\xi-2 \xi^{0}\right|_{\infty} \leq \varepsilon / 2\right\}
\end{aligned}
$$

Then, for $\xi \in \Pi_{\xi} \operatorname{supp}\left(V_{\phi_{\varepsilon}}\left(M_{2 \xi^{0}} \psi_{\varepsilon}\right)\right)$ we have $|\xi|_{\infty} \geq 2\left|\xi^{0}\right|_{\infty}-\varepsilon / 2$. Hence, for all $\xi \in \mathbb{R}^{d} \backslash\{0\}$ :

$$
\begin{aligned}
\left\|e^{-\lambda \omega\left(\frac{x}{N+1}\right)-\mu \omega(\xi)}|\xi|_{\infty}^{-N} V_{\phi_{\varepsilon}}\left(M_{2 \xi^{0}} \psi_{\varepsilon}\right)(x, \xi)\right\|_{L^{p^{\prime}, q^{\prime}}} & \leq\left(2\left|\xi^{0}\right|_{\infty}-\frac{\varepsilon}{2}\right)^{-N}\left\|V_{\phi_{\varepsilon}}\left(M_{2 \xi^{0}} \psi_{\varepsilon}\right)\right\|_{L^{p^{\prime}, q^{\prime}}} \\
& \leq C_{\varepsilon, \xi^{0}}\left(2\left|\xi^{0}\right|_{\infty}-\frac{\varepsilon}{2}\right)^{-N}
\end{aligned}
$$

for some $C_{\varepsilon, \xi^{0}}>0$, since $V_{\phi_{\varepsilon}}\left(M_{2 \xi^{0}} \psi_{\varepsilon}\right) \in \mathcal{S}_{\omega}\left(\mathbb{R}^{2 d}\right) \subseteq L^{p^{\prime}, q^{\prime}}\left(\mathbb{R}^{2 d}\right)$, where we have denoted by $p^{\prime}, q^{\prime}$ the conjugate exponents of $p$ and $q$ respectively.

On the other hand, for $\xi \in \mathbb{R}^{d} \backslash\{0\}$, by (3.29):

$$
\begin{aligned}
& \left(2\left|\xi^{0}\right|_{\infty}-\varepsilon\right)^{N}\left|\left\langle V_{\tilde{f}} f(x, \xi), e^{-i\left\langle\xi^{0}, x\right\rangle} V_{\phi_{\varepsilon}}\left(M_{2 \xi^{0}} \psi_{\varepsilon}\right)(x, \xi)\right\rangle\right| \\
& \leq\left(2\left|\xi^{0}\right|_{\infty}-\varepsilon\right)^{N}\left|\left\langle e^{\lambda \omega\left(\frac{x}{N+1}\right)+\mu \omega(\xi)}|\xi|_{\infty}^{N} V_{\tilde{f}} f(x, \xi), e^{-\lambda \omega\left(\frac{x}{N+1}\right)-\mu \omega(\xi)}|\xi|_{\infty}^{-N} e^{-i\left\langle\xi^{0}, x\right\rangle} V_{\phi_{\varepsilon}}\left(M_{2 \xi^{0}} \psi_{\varepsilon}\right)(x, \xi)\right\rangle\right| \\
& \leq\left(2\left|\xi^{0}\right|_{\infty}-\varepsilon\right)^{N}\left\|\left.e^{\lambda \omega\left(\frac{x}{N+1}\right)+\mu \omega(\xi)}|\xi|_{\infty}^{N} V_{\tilde{f}} f\right|_{L^{p, q}} \cdot\right\| e^{-\lambda \omega\left(\frac{x}{N+1}\right)-\mu \omega(\xi)}|\xi|_{\infty}^{-N} V_{\phi_{\varepsilon}}\left(M_{2 \xi^{0}} \psi_{\varepsilon}\right) \|_{L^{p^{\prime}, q^{\prime}}} \\
& \leq C_{\varepsilon, \xi^{0}} \frac{\left(2\left|\xi^{0}\right|_{\infty}-\varepsilon\right)^{N}}{\left(2\left|\xi^{0}\right|_{\infty}-\varepsilon / 2\right)^{N}}\left\|e^{\lambda \omega\left(\frac{x}{N+1}\right)+\mu \omega(\xi)}|\xi|_{\infty}^{N} V_{\tilde{f}} f\right\|_{L^{p, q}} \leq C_{\varepsilon, \xi^{0}}\left\|e^{\lambda \omega\left(\frac{x}{N+1}\right)+\mu \omega(\xi)}|\xi|_{\infty}^{N} V_{\tilde{f}} f\right\|_{L^{p, q}} .
\end{aligned}
$$

Then

$$
\begin{array}{r}
\left(2\left|\xi^{0}\right|_{\infty}-\varepsilon\right)\left|\left\langle V_{\tilde{f}} f(x, \xi), e^{-i\left\langle\xi^{0}, x\right\rangle} V_{\phi_{\varepsilon}}\left(M_{2 \xi^{0}} \psi_{\varepsilon}\right)(x, \xi)\right\rangle\right|^{\frac{1}{N}} \leq \\
\leq C_{\varepsilon, \xi^{0}}^{\frac{1}{N}}\left\|e^{\lambda \omega\left(\frac{x}{N+1}\right)+\mu \omega(\xi)}|\xi|_{\infty}^{N} V_{\tilde{f}} f\right\|_{L^{p, q}}^{\frac{1}{N}}
\end{array}
$$


Let us now remark that

$$
\begin{aligned}
V_{\phi_{\varepsilon}}\left(M_{2 \xi^{0}} \psi_{\varepsilon}\right)(x, \xi) & =\int_{\mathbb{R}^{d}} e^{i\left\langle 2 \xi^{0}, y\right\rangle} \psi_{\varepsilon}(y) \overline{\phi_{\varepsilon}(y-x)} e^{-i\langle y, \xi\rangle} d y \\
& =e^{i\left\langle\xi^{0}, x\right\rangle} \int_{\mathbb{R}^{d}} e^{i\left\langle\xi^{0}, y\right\rangle} \psi_{\varepsilon}(y) \overline{e^{-i\left\langle\xi^{0}, y-x\right\rangle} \phi_{\varepsilon}(y-x)} e^{-i\langle y, \xi\rangle} d y \\
& =e^{i\left\langle\xi^{0}, x\right\rangle} V_{M_{-\xi^{0}} \phi_{\varepsilon}}\left(M_{\xi^{0}} \psi_{\varepsilon}\right)(x, \xi)
\end{aligned}
$$

and therefore, from [18, Thm. 3.2.1], by (3.28):

$$
\begin{aligned}
& \left\langle V_{\tilde{f}} f(x, \xi), e^{-i\left\langle\xi^{0}, x\right\rangle} V_{\phi_{\varepsilon}}\left(M_{2 \xi^{0}} \psi_{\varepsilon}\right)(x, \xi)\right\rangle=\left\langle V_{\tilde{f}} f, V_{M_{-\xi^{0}} \phi_{\varepsilon}}\left(M_{\xi^{0}} \psi_{\varepsilon}\right)\right\rangle \\
& =\left\langle f, M_{\xi^{0}} \psi_{\varepsilon}\right\rangle \cdot \overline{\left\langle\tilde{f}, M_{-\xi^{0}} \phi_{\varepsilon}\right\rangle}=\left\langle\widehat{f}, \widehat{M_{\xi^{0}} \psi_{\varepsilon}}\right\rangle \cdot \widehat{\left\langle\widehat{\tilde{f}}, \widehat{M_{-\xi^{0}} \phi_{\varepsilon}}\right\rangle} \\
& \left.\left.=\left\langle\widehat{f}, T_{\xi^{0}} \widehat{\psi}_{\varepsilon}\right\rangle \cdot \overline{\left\langle\tilde{\tilde{f}}, T_{-\xi^{0}} \widehat{\phi}_{\varepsilon}\right.}\right\rangle=\left\langle\widehat{f}, T_{\xi^{0}} \widehat{\psi_{\varepsilon}}\right\rangle \cdot \overline{\left\langle\widehat{f}, T_{\xi^{0}} \widehat{\tilde{\phi}}_{\varepsilon}\right.}\right\rangle=0 .
\end{aligned}
$$

Therefore

$$
\lim _{N \rightarrow+\infty}\left|\left\langle V_{\tilde{f}} f(x, \xi), e^{-i\left\langle\xi^{0}, x\right\rangle} V_{\phi_{\varepsilon}}\left(M_{2 \xi^{0}} \psi_{\varepsilon}\right)(x, \xi)\right\rangle\right|^{1 / N}=1
$$

and from (3.30) we obtain that

$$
\left(2\left|\xi^{0}\right|_{\infty}-\varepsilon\right) \leq \liminf _{N \rightarrow+\infty}\left\|e^{\lambda \omega\left(\frac{x}{N+1}\right)+\mu \omega(\xi)}|\xi|_{\infty}^{N} V_{\tilde{f}} f\right\|_{L^{p, q}}^{1 / N}
$$

By the arbitrariness of $0<\varepsilon<2\left|\xi^{0}\right|_{\infty}$ and of $\xi^{0} \in \operatorname{supp} \hat{f}$, from (3.31) and (3.27), we finally obtain (3.26).

Corollary 3.14. Let $f \in \mathcal{S}_{\omega}\left(\mathbb{R}^{d}\right)$ and $p, q \in[1,+\infty]$. Then, for all $\lambda, \mu \geq 0$ :

$$
\lim _{N \rightarrow+\infty}\left\|e^{\lambda \omega\left(\frac{x}{N+1}\right)+\mu \omega(\xi)}|\xi|_{\infty}^{N} \operatorname{Wig} f(x, \xi)\right\|_{L^{p, q}}^{1 / N}=R_{\hat{f}}
$$

Proof. By [18, Lemma 4.3.1], if $p, q \in[1,+\infty)$ :

$$
\begin{aligned}
& \left\|e^{\lambda \omega\left(\frac{x}{N+1}\right)+\mu \omega(\xi)}|\xi|_{\infty}^{N} \operatorname{Wig} f(x, \xi)\right\|_{L^{p, q}} \\
& =\left\|e^{\lambda \omega\left(\frac{x}{N+1}\right)+\mu \omega(\xi)}|\xi|_{\infty}^{N} 2^{d} e^{2 i\langle x, \xi\rangle} V_{\tilde{f}} f(2 x, 2 \xi)\right\|_{L^{p, q}} \\
& =2^{d} 2^{-\frac{d}{p}} 2^{-\frac{d}{q}}\left\|e^{\lambda \omega\left(\frac{y}{2(N+1)}\right)+\mu \omega\left(\frac{\eta}{2}\right)}\left|\frac{\eta}{2}\right|_{\infty}^{N} V_{\tilde{f}} f(y, \eta)\right\|_{L^{p, q}}
\end{aligned}
$$

Using the fact that $\omega$ is increasing and satisfies condition $(\alpha)$ of Definition 2.1 we have

$$
\frac{1}{L} \omega(t)-1 \leq \omega\left(\frac{t}{2}\right) \leq \omega(t),
$$


and so, by (3.33),

$$
\begin{aligned}
2^{\frac{d}{N}\left(1-\frac{1}{p}-\frac{1}{q}\right)} \frac{1}{2} e^{-\lambda / N-\mu / N}\left\|e^{\frac{\lambda}{L} \omega\left(\frac{y}{N+1}\right)+\frac{\mu}{L} \omega(\eta)}|\eta|_{\infty}^{N} V_{\tilde{f}} f(y, \eta)\right\|_{L^{p, q}}^{1 / N} \\
\leq\left\|e^{\lambda \omega\left(\frac{x}{N+1}\right)+\mu \omega(\xi)}|\xi|_{\infty}^{N} \operatorname{Wig} f(x, \xi)\right\|_{L^{p, q}}^{1 / N} \\
\leq 2^{\frac{d}{N}\left(1-\frac{1}{p}-\frac{1}{q}\right)} \frac{1}{2}\left\|e^{\lambda \omega\left(\frac{y}{N+1}\right)+\mu \omega(\eta)}|\eta|_{\infty}^{N} V_{\tilde{f}} f(y, \eta)\right\|_{L^{p, q}}^{1 / N}
\end{aligned}
$$

Consequently, from Proposition 3.13, we deduce

$$
\lim _{N \rightarrow+\infty}\left\|e^{\lambda \omega\left(\frac{x}{N+1}\right)+\mu \omega(\xi)}|\xi|_{\infty}^{N} \operatorname{Wig} f(x, \xi)\right\|_{L^{p, q}}^{1 / N}=\frac{1}{2} \cdot 2 R_{\hat{f}}=R_{\hat{f}}
$$

for $1 \leq p, q<\infty$. If $p$ and/or $q$ is $\infty$ the proof is similar.

Corollary 3.15. Let $f \in \mathcal{S}_{\omega}\left(\mathbb{R}^{d}\right)$ and $p, q \in[1,+\infty]$. Then

$$
\begin{aligned}
& \lim _{N \rightarrow+\infty}\left\||\xi|_{\infty}^{N} \operatorname{Wig} f(x, \xi)\right\|_{L^{p, q}}^{1 / N}=R_{\hat{f}} \\
& \lim _{N \rightarrow+\infty}\left\||x|_{\infty}^{N} \operatorname{Wig} f(x, \xi)\right\|_{L^{p, q}}^{1 / N}=R_{f} .
\end{aligned}
$$

Proof. Formula (3.34) follows from (3.32) with $\lambda=\mu=0$.

Formula (3.35) follows from [18, Prop. 4.3.2] and (3.34) applied to $\hat{f}$ :

$$
\begin{aligned}
\lim _{N \rightarrow+\infty}\left\||x|_{\infty}^{N} \operatorname{Wig} f(x, \xi)\right\|_{L^{p, q}}^{1 / N} & =\lim _{N \rightarrow+\infty}\left\||x|_{\infty}^{N} \operatorname{Wig} \hat{f}(\xi,-x)\right\|_{L^{p, q}}^{1 / N} \\
& =\lim _{N \rightarrow+\infty}\left\||x|_{\infty}^{N} \operatorname{Wig} \hat{f}(\xi, x)\right\|_{L^{p, q}}^{1 / N}=R_{\hat{\hat{f}}}=R_{f} .
\end{aligned}
$$

If we consider formula (3.34) for $p=q=2$ in the one-dimensional case, the multiplication by $|\xi|^{N}$ cannot be replaced by the derivatives $D_{x}^{N}$ of the Wigner transform of a real valued function $f \in \mathcal{S}_{\omega}(\mathbb{R})$. Indeed, if we denote by

$$
A f(x, \xi):=\int_{\mathbb{R}} f\left(t+\frac{x}{2}\right) \overline{f\left(t-\frac{x}{2}\right)} e^{-i\langle t, \xi\rangle} d t
$$

the ambiguity function $A f$ of $f$, by [18, Lemma 4.3.4], we obtain

$$
\begin{aligned}
\left\|D_{x}^{N} \operatorname{Wig} f(x, \xi)\right\|_{L^{2}} & =\left\|\mathcal{F}\left(D_{x}^{N} \operatorname{Wig} f(x, \xi)\right)\right\|_{L^{2}} \\
& =\left\|y^{N} \widehat{\operatorname{Wig} f}(y, \eta)\right\|_{L^{2}}=\left\|y^{N} A f(-\eta, y)\right\|_{L^{2}} .
\end{aligned}
$$

Now, since $f$ is real valued by assumption,

$$
\begin{aligned}
A f(-\eta, y) & =\int_{\mathbb{R}} f\left(t-\frac{\eta}{2}\right) \overline{f\left(t+\frac{\eta}{2}\right)} e^{-i\langle t, y\rangle} d t \\
& =\int_{\mathbb{R}} f(u-\eta) f(u) e^{-i\left\langle u-\frac{\eta}{2}, y\right\rangle} d u=e^{\frac{i}{2}\langle\eta, y\rangle} V_{f} f(\eta, y) .
\end{aligned}
$$

Hence, by (3.36),

$$
\limsup _{N \rightarrow+\infty}\left\|D_{x}^{N} \operatorname{Wig} f(x, \xi)\right\|_{L^{2}}^{1 / N}=\limsup _{N \rightarrow+\infty}\left\|y^{N} V_{f} f(\eta, y)\right\|_{L^{2}}^{1 / N},
$$


which can be strictly smaller than $R_{\hat{f}}$, by Example 3.12 .

On the other hand, if $f=\tilde{f}$, by Proposition 3.13,

$$
\lim _{N \rightarrow+\infty}\left\|D_{x}^{N} \operatorname{Wig} f(x, \xi)\right\|_{L^{2}}^{1 / N}=\lim _{N \rightarrow+\infty}\left\|y^{N} V_{\tilde{f}} f(\eta, y)\right\|_{L^{2}}^{1 / N}=2 R_{\hat{f}}>R_{\hat{f}} .
$$

Lemma 3.16. Let $p, q \in[1,+\infty]$ and $f \in \mathcal{S}_{\omega}^{\prime}\left(\mathbb{R}^{d}\right)$ such that

$$
e^{\lambda \omega(x)+\mu \omega(\xi)} \operatorname{Wig} f(x, \xi) \in L^{p, q}\left(\mathbb{R}^{2 d}\right)
$$

for all $\lambda, \mu>0$. Then $f \in \mathcal{S}_{\omega}\left(\mathbb{R}^{d}\right)$.

Proof. We observe that Wig $f \in L^{1}\left(\mathbb{R}^{2 d}\right)$, since

$$
\|\operatorname{Wig} f\|_{L^{1}} \leq\left\|e^{\lambda \omega(x)+\mu \omega(\xi)} \operatorname{Wig} f(x, \xi)\right\|_{L^{p, q}}\left\|e^{-\lambda \omega(x)-\mu \omega(\xi)}\right\|_{L^{p^{\prime}, q^{\prime}}}<\infty
$$

by hypothesis and (2.3), provided that $\lambda \geq(d+1) / b p^{\prime}$ and $\mu \geq(d+1) / b q^{\prime}$. Then, by applying the inverse partial Fourier transform with respect to $\xi$ to $\operatorname{Wig} f(x, \xi)$ we get

$$
f\left(x+\frac{t}{2}\right) \overline{f\left(x-\frac{t}{2}\right)}=(2 \pi)^{-d} \int_{\mathbb{R}^{d}} \operatorname{Wig} f(x, \xi) e^{i\langle\xi, t\rangle} d \xi .
$$

Then, the element $f\left(x+\frac{t}{2}\right) \overline{f\left(x-\frac{t}{2}\right)}$, that a priori belongs to $\mathcal{S}_{\omega}^{\prime}\left(\mathbb{R}_{(t, x)}^{2 d}\right)$, is in fact a function in $L^{\infty}\left(\mathbb{R}_{t}^{d}\right)$ for almost every $x \in \mathbb{R}^{d}$, and is in $L^{1}\left(\mathbb{R}_{x}^{d}\right)$ for every $t \in \mathbb{R}^{d}$. Now, suppose that $f \not \equiv 0$ (otherwise the result is trivial), and let $\phi_{0} \in \mathcal{S}_{\omega}\left(\mathbb{R}^{d}\right)$ such that $\left\langle\bar{f}, \phi_{0}\right\rangle \neq 0$. For a function $\phi \in \mathcal{S}_{\omega}\left(\mathbb{R}^{d}\right)$, consider

$$
\Phi(t, x)=\phi\left(x+\frac{t}{2}\right) \phi_{0}\left(x-\frac{t}{2}\right) \in \mathcal{S}_{\omega}\left(\mathbb{R}^{2 d}\right),
$$

and apply the two distributions in (3.37) to the test function $\Phi$; on the right-hand side we can write the application as an integral, and then we obtain

$$
\langle f, \phi\rangle\left\langle\bar{f}, \phi_{0}\right\rangle=(2 \pi)^{-d} \int_{\mathbb{R}^{2 d}}\left(\int_{\mathbb{R}^{d}} \operatorname{Wig} f(x, \xi) e^{i\langle\xi, t\rangle} d \xi\right) \phi\left(x+\frac{t}{2}\right) \phi_{0}\left(x-\frac{t}{2}\right) d x d t .
$$

Then by the change of variables $x+t / 2=y, x-t / 2=s$ and by Fubini Theorem we obtain

$$
\langle f, \phi\rangle=\frac{1}{(2 \pi)^{d}\left\langle\bar{f}, \phi_{0}\right\rangle} \int_{\mathbb{R}^{d}}\left(\int_{\mathbb{R}^{2 d}} \operatorname{Wig} f\left(\frac{y+s}{2}, \xi\right) e^{i\langle\xi, y-s\rangle} \phi_{0}(s) d \xi d s\right) \phi(y) d y,
$$

and so we get that $f$ is a function in $L^{1}\left(\mathbb{R}^{d}\right)$ given by

$$
f(x)=\frac{1}{(2 \pi)^{d}\left\langle\bar{f}, \phi_{0}\right\rangle} \int_{\mathbb{R}^{2 d}} \operatorname{Wig} f\left(\frac{x+s}{2}, \xi\right) e^{i\langle\xi, x-s\rangle} \phi_{0}(s) d \xi d s .
$$

In order to prove that $f \in \mathcal{S}_{\omega}\left(\mathbb{R}^{d}\right)$ we shall prove that $f$ satisfies condition $(c)^{\prime}$ of Theorem 2.5. Suppose that $p<+\infty$. By (3.38) and Minkowski inequality, cf. for example [17, 6.19], we have

$$
\begin{aligned}
\left\|e^{\lambda \omega(x)} f(x)\right\|_{L^{p}} & \leq \frac{1}{(2 \pi)^{d}\left|\left\langle\bar{f}, \phi_{0}\right\rangle\right|}\left(\int_{\mathbb{R}^{d}}\left(\int_{\mathbb{R}^{2 d}} e^{\lambda \omega(x)}\left|\operatorname{Wig} f\left(\frac{x+s}{2}, \xi\right)\right|\left|\phi_{0}(s)\right| d \xi d s\right)^{p} d x\right)^{1 / p} \\
& \leq \frac{1}{(2 \pi)^{d}\left|\left\langle\bar{f}, \phi_{0}\right\rangle\right|} \int_{\mathbb{R}^{2 d}}\left(\int_{\mathbb{R}^{d}}\left(e^{\lambda \omega(x)}\left|\operatorname{Wig} f\left(\frac{x+s}{2}, \xi\right)\right|\left|\phi_{0}(s)\right|\right)^{p} d x\right)^{1 / p} d \xi d s .
\end{aligned}
$$


Writing $C_{0}=\left((2 \pi)^{d}\left|\left\langle\bar{f}, \phi_{0}\right\rangle\right|\right)^{-1}$, using Hölder inequality in the $\xi$-integral and (2.1) we obtain, for $\mu \geq(d+1) / b q^{\prime}$,

$$
\begin{aligned}
\left\|e^{\lambda \omega(x)} f(x)\right\|_{L^{p}} \leq & C_{0} e^{\lambda L} \int_{\mathbb{R}^{2 d}} e^{-\mu \omega(\xi)} e^{\mu \omega(\xi)} \\
& \cdot\left(\int_{\mathbb{R}^{d}}\left(e^{\lambda L \omega(x+s)}\left|\operatorname{Wig} f\left(\frac{x+s}{2}, \xi\right)\right|\right)^{p} d x\right)^{1 / p} e^{\lambda L \omega(s)}\left|\phi_{0}(s)\right| d \xi d s \\
\leq & C_{0} 2^{d / p} e^{\lambda\left(L^{2}+L\right)}\left\|e^{-\mu \omega(\xi)}\right\|_{L^{q^{\prime}}} \\
& \cdot\left(\int_{\mathbb{R}^{d}} e^{\lambda L \omega(s)}\left|\phi_{0}(s)\right| d s\right)\left\|e^{\mu \omega(\xi)}\left(\int_{\mathbb{R}^{d}}\left(e^{\lambda L^{2} \omega(y)}|\mathrm{Wig} f(y, \xi)|\right)^{p} d y\right)^{1 / p}\right\|_{L^{q}\left(\mathbb{R}_{\xi}^{d}\right)} \\
= & C_{\lambda}\left\|e^{\lambda L^{2} \omega(y)+\mu \omega(\xi)} \operatorname{Wig} f(y, \xi)\right\|_{L^{p . q}}<\infty
\end{aligned}
$$

by hypothesis and (2.3). In the case $p=+\infty$ the same proof works, with small modifications, so (3.39) holds for every $p$ and $q$.

Now, let $\phi_{1} \in \mathcal{S}_{\omega}\left(\mathbb{R}^{d}\right)$ be such that $\left\langle\overline{\hat{f}}, \phi_{1}\right\rangle \neq 0$, and $q<+\infty$. We apply (3.38) to $\hat{f}$ and use [18, Prop. 4.3.2] to get

$$
\begin{aligned}
\left\|e^{\lambda \omega(\xi)} \hat{f}(\xi)\right\|_{L^{q}} & \leq C_{1}\left(\int_{\mathbb{R}^{d}}\left(e^{\lambda \omega(\xi)} \int_{\mathbb{R}^{2 d}}\left|\operatorname{Wig} \hat{f}\left(\frac{\xi+s}{2}, y\right)\right|\left|\phi_{1}(s)\right| d y d s\right)^{q} d \xi\right)^{1 / q} \\
& =C_{1}\left(\int_{\mathbb{R}^{d}}\left(e^{\lambda \omega(\xi)} \int_{\mathbb{R}^{2 d}}\left|\operatorname{Wig} f\left(-y, \frac{\xi+s}{2}\right)\right|\left|\phi_{1}(s)\right| d y d s\right)^{q} d \xi\right)^{1 / q},
\end{aligned}
$$

where $C_{1}=\left((2 \pi)^{d}\left|\left\langle\overline{\hat{f}}, \phi_{1}\right\rangle\right|\right)^{-1}$. We apply the change of variables $(\xi+s) / 2=\eta$ in the $\xi$-integral, $-y=x$, use condition $(\alpha)$ of Definition 2.1 and (2.1) and Hölder's inequality in the $x$-integral to obtain, for $\mu \geq(d+1) / b p^{\prime}$,

$$
\begin{aligned}
\left\|e^{\lambda \omega(\xi)} \hat{f}(\xi)\right\|_{L^{q}} \leq & C_{1} 2^{\frac{d}{q}} e^{\lambda\left(L^{2}+L\right)} \int_{\mathbb{R}^{d}} e^{\lambda L \omega(s)}\left|\phi_{1}(s)\right| d s \\
& \cdot\left(\int_{\mathbb{R}^{d}}\left(e^{\lambda L^{2} \omega(\eta)} \int_{\mathbb{R}^{d}} e^{-\mu \omega(x)} e^{\mu \omega(x)}|\operatorname{Wig} f(x, \eta)| d x\right)^{q} d \eta\right)^{1 / q} \\
\leq & C_{\lambda}\left(\int_{\mathbb{R}^{d}}\left(\int_{\mathbb{R}^{d}}\left(e^{\lambda L^{2} \omega(\eta)} e^{\mu \omega(x)}|\operatorname{Wig} f(x, \eta)|\right)^{p} d x\right)^{q / p} d \eta\right)^{1 / q} \\
= & C_{\lambda}\left\|e^{\mu \omega(x)+\lambda L^{2} \omega(\eta)} \operatorname{Wig} f(x, \eta)\right\|_{L^{p, q}}<\infty
\end{aligned}
$$

by hypothesis, where $C_{\lambda}=C_{1} 2^{d / q} e^{\lambda\left(L^{2}+L\right)} \int e^{\lambda L \omega(s)}\left|\phi_{1}(s)\right| d s\left\|e^{-\mu \omega(x)}\right\|_{L^{p^{\prime}}}<+\infty$ by (2.3). If $q=\infty$ the same proof works, with small modifications, so (3.40) holds for every $p$ and $q$. By (3.39) and (3.40) the function $f$ satisfies Theorem $2.5(c)^{\prime}$.

We can now prove the following theorem that, besides the classical result in ultradifferentiable classes (see $[6,14,16]$ ), contains real ultradifferentiable Paley-Wiener theorems in the spirit of [3] and a new equivalent condition on the Wigner transform. Given $R>0$, for the compact set 
$Q_{R}$, as defined in (3.3), we denote its supporting function by

$$
H_{R}(x):=\sup \left\{\langle x, y\rangle: y \in \mathbb{R}^{d},|y|_{\infty} \leq R\right\} .
$$

Theorem 3.17. Let $1 \leq p, q \leq+\infty, R>0$ and $f \in C^{\infty}\left(\mathbb{R}^{d}\right)$. Then the following conditions are equivalent:

(a) $f$ is an entire function in $\mathbb{C}^{d}$ and for all $k \in \mathbb{N}_{0}$ there exists $C_{k}>0$ such that

$$
|f(z)| \leq C_{k} e^{H_{R}(\operatorname{Im} z)-k \omega(z)}, \quad z \in \mathbb{C}^{d} .
$$

(b) $f \in \mathrm{PW}_{R}^{\omega}\left(\mathbb{R}^{d}\right)$.

(c) $e^{\lambda \omega(x)} f^{(\alpha)}(x) \in L^{p}\left(\mathbb{R}^{d}\right)$ for all $\alpha \in \mathbb{N}_{0}^{d}$ and $\lambda \geq 0$ and

$$
\lim _{n \rightarrow+\infty}\left(\max _{|\alpha|=n}\left\|e^{\lambda \omega\left(\frac{x}{n+1}\right)} f^{(\alpha)}(x)\right\|_{L^{p}}\right)^{1 / n}=R_{\hat{f}} \leq R .
$$

(d) $f \in \mathcal{S}_{\omega}\left(\mathbb{R}^{d}\right)$ and $\operatorname{supp} \hat{f} \subseteq Q_{R}$.

(e) $f \in \mathcal{S}_{\omega}^{\prime}\left(\mathbb{R}^{d}\right)$, $e^{\lambda \omega(x)+\mu \omega(\xi)} \operatorname{Wig} f(x, \xi) \in L^{p, q}\left(\mathbb{R}^{2 d}\right)$ for all $\lambda, \mu \geq 0$ and

$$
\lim _{n \rightarrow+\infty}\left\|e^{\lambda \omega\left(\frac{x}{n+1}\right)+\mu \omega(\xi)}|\xi|_{\infty}^{n} \operatorname{Wig} f(x, \xi)\right\|_{L^{p, q}}^{1 / n}=R_{\hat{f}} \leq R .
$$

Proof. $(a) \Leftrightarrow(d)$ : This is Paley-Wiener theorem in $\mathcal{D}_{(\omega)}\left(\mathbb{R}^{d}\right)$ (Beurling case) for the convex set $Q_{R}$; see [7, Theorem 2.14], or [6, Theorem 1.4.1] and [16, Satz 3.3], or [14, Lemma 3.3] when the non-quasianalytic weight $\omega$ satisfies the additional assumption $\log (1+t)=o(\omega(t))$ as $t \rightarrow+\infty$.

$(d) \Leftrightarrow(b)$ : This is Theorem 3.2.

$(d) \Rightarrow(c)$ : It follows from Theorem 2.5( $a)^{\prime}$, Proposition 3.3 and Remark 3.5.

$(c) \Rightarrow(d)$ : It follows from Proposition 3.3, Remark 3.5 and Lemma 3.8 .

$(d) \Rightarrow(e)$ : It is Corollary 3.14, since for $f \in \mathcal{S}_{\omega}\left(\mathbb{R}^{d}\right)$,

$$
e^{\lambda \omega(x)+\mu \omega(\xi)} \operatorname{Wig} f(x, \xi) \in L^{p, q}\left(\mathbb{R}^{2 d}\right)
$$

for all $\lambda, \mu \geq 0$.

$(e) \Rightarrow(d)$ : Follows from Lemma 3.16 and Corollary 3.14.

Corollary 3.18. Given $1 \leq p, q \leq+\infty$ and $R>0$, we consider $f \in \mathcal{S}_{\omega}^{\prime}\left(\mathbb{R}^{d}\right)$ such that $e^{\lambda \omega(x)+\mu \omega(\xi)}$ Wig $f \in L^{p, q}\left(\mathbb{R}^{2 d}\right)$ for all $\lambda, \mu \geq 0$. We have:

(a) $f \in \mathcal{S}_{\omega}\left(\mathbb{R}^{d}\right)$ with supp $\hat{f} \subseteq Q_{R}$ if and only if $R_{\hat{f}} \leq R$ and for all $\lambda, \mu>0$ there exists $C_{\lambda, \mu}>0$ such that

$$
|\xi|_{\infty}^{n} \operatorname{Wig} f(x, \xi)<C_{\lambda, \mu} R^{n}(n+1)^{\frac{d}{2}} e^{-\lambda \omega\left(\frac{x}{n+1}\right)-\mu \omega(\xi)}, \quad n \in \mathbb{N}_{0}, \quad(x, \xi) \in \mathbb{R}^{2 d} .
$$

(b) $f \in \mathcal{S}_{\omega}\left(\mathbb{R}^{d}\right)$ with supp $f \subseteq Q_{R}$ if and only if $R_{f} \leq R$ and for all $\lambda, \mu>0$ there exists $C_{\lambda, \mu}>0$ such that

$$
|x|_{\infty}|\operatorname{Wig} f(x, \xi)| \leq C_{\mu, \lambda} R^{n}(n+1)^{\frac{d}{2}} e^{-\lambda \omega\left(\frac{\xi}{n+1}\right)-\mu \omega(x)}, \quad n \in \mathbb{N}_{0}, \quad(x, \xi) \in \mathbb{R}^{2 d} .
$$

Proof. (a) If $f \in \mathcal{S}_{\omega}\left(\mathbb{R}^{d}\right)$ and supp $\hat{f} \subseteq Q_{R}$, by Theorem 3.17, we obtain that $f \in \mathrm{PW}_{R}^{\omega}\left(\mathbb{R}^{d}\right)$. From Proposition 3.10 we have $f \in \mathrm{PWG}_{R}^{\omega, \tilde{f}}$, where $\tilde{f}(x)=f(-x)$, and hence

$$
\sup _{n \in \mathbb{N}_{0}} \sup _{x, \xi \in \mathbb{R}^{d}}(2 R)^{-n} \frac{1}{(n+1)^{d / 2}} e^{\lambda \omega\left(\frac{x}{n+1}\right)+\mu \omega(\xi)}|\xi|_{\infty}^{n}\left|V_{\tilde{f}} f(x, \xi)\right|<+\infty .
$$


It follows from [18, Lemma 4.3.1] that

$$
\begin{aligned}
& \sup _{n \in \mathbb{N}_{0}} \sup _{x, \xi \in \mathbb{R}^{d}} R^{-n} \frac{1}{(n+1)^{d / 2}} e^{\lambda \omega\left(\frac{x}{n+1}\right)+\mu \omega(\xi)}|\xi|_{\infty}^{n}|\operatorname{Wig} f(x, \xi)| \\
& \leq \sup _{n \in \mathbb{N}_{0}} \sup _{x, \xi \in \mathbb{R}^{d}} R^{-n} \frac{1}{(n+1)^{d / 2}} e^{\lambda \omega\left(\frac{2 x}{n+1}\right)+\mu \omega(2 \xi)} \frac{|2 \xi|_{\infty}^{n}}{2^{n}}\left|2^{d} V_{\tilde{f}} f(2 x, 2 \xi)\right| \\
& =2^{d} \sup _{n \in \mathbb{N}_{0}} \sup _{x, \xi \in \mathbb{R}^{d}}(2 R)^{-n} \frac{1}{(n+1)^{d / 2}} e^{\lambda \omega\left(\frac{x}{n+1}\right)+\mu \omega(\xi)}|\xi|_{\infty}^{n}\left|V_{\tilde{f}} f(x, \xi)\right|<+\infty .
\end{aligned}
$$

Conversely, if $f \in \mathcal{S}_{\omega}^{\prime}\left(\mathbb{R}^{d}\right)$ with $e^{\lambda \omega(x)+\mu \omega(\xi)}$ Wig $f \in L^{p, q}\left(\mathbb{R}^{2 d}\right)$ and the inequality of (a) is satisfied, then $f \in \mathcal{S}_{\omega}\left(\mathbb{R}^{d}\right)$ by Lemma 3.16 and supp $\hat{f} \subseteq Q_{R}$ by Corollary 3.14, since $R_{\hat{f}} \leq R$.

(b) It follows from (a) because

$$
\sup _{n \in \mathbb{N}_{0}} \sup _{(x, \xi) \in \mathbb{R}^{2 d}} R^{-n} \frac{1}{(n+1)^{\frac{d}{2}}} e^{\lambda \omega\left(\frac{x}{n+1}\right)+\mu \omega(\xi)}|\xi|_{\infty}^{n}|\operatorname{Wig} \hat{f}(x, \xi)|<+\infty
$$

is equivalent to

$$
\sup _{n \in \mathbb{N}_{0}} \sup _{(x, \xi) \in \mathbb{R}^{2 d}} R^{-n} \frac{1}{(n+1)^{\frac{d}{2}}} e^{\lambda \omega\left(\frac{\xi}{n+1}\right)+\mu \omega(x)}|x|_{\infty}^{n}|\operatorname{Wig} f(x, \xi)|<+\infty,
$$

since Wig $\hat{f}(x, \xi)=\operatorname{Wig} f(-\xi, x)$ by [18, Prop. 4.3.2].

If we consider $\omega(t)=\log (1+t)$ we have that $\mathcal{S}_{\omega}\left(\mathbb{R}^{d}\right)$ is the classical Schwartz space $\mathcal{S}\left(\mathbb{R}^{d}\right)$ and hence Theorem 3.2 with $d=1$ coincides with Theorem 1 of [2], while Proposition 3.3 for $d=1$ and $\lambda=0$ coincides with Theorem 1 of [5]. We also observe that Lemma 3.6 for $\omega(t)=\log (1+t)$ implies

$f \in C^{\infty}\left(\mathbb{R}^{d}\right),(1+|x|)^{\lambda} f^{(\alpha)}(x) \in L^{p}\left(\mathbb{R}^{d}\right) \forall \lambda>0, \forall \alpha \in \mathbb{N}_{0}^{d}$, for some $p \in[1,+\infty] \Leftrightarrow f \in \mathcal{S}\left(\mathbb{R}^{d}\right)$. Moreover, Lemma 3.16 for $\omega(t)=\log (1+t)$ implies

$$
\begin{aligned}
& f \in \mathcal{S}^{\prime}\left(\mathbb{R}^{d}\right),(1+|x|)^{\lambda}(1+|\xi|)^{\mu} \operatorname{Wig} f(x, \xi) \in L^{p, q}\left(\mathbb{R}^{d}\right) \forall \mu, \lambda>0, \text { for some } p, q \in[1,+\infty] \\
& \Leftrightarrow f \in \mathcal{S}\left(\mathbb{R}^{d}\right) .
\end{aligned}
$$

The above remarks lead to the following corollary of Theorem 3.17 for $\omega(t)=\log (1+t)$ :

Corollary 3.19. Let $1 \leq p, q \leq+\infty, R>0$ and $f \in C^{\infty}\left(\mathbb{R}^{d}\right)$. Then the following conditions are equivalent:

(a) $f$ is an entire function in $\mathbb{C}^{d}$ and for all $k \in \mathbb{N}_{0}$ there exists $C_{k}>0$ such that

$$
|f(z)| \leq C_{k}(1+|z|)^{-k} e^{H_{R}(\operatorname{Im} z)}, \quad z \in \mathbb{C}^{d} .
$$

(b) $f \in \mathcal{S}\left(\mathbb{R}^{d}\right)$ and for all $\lambda>0$ there exists $C_{\lambda}>0$ such that

$$
\left|f^{(\alpha)}(x)\right| \leq C_{\lambda} R^{|\alpha|}(|\alpha|+1)^{\lambda}(1+|x|)^{-\lambda} \quad x \in \mathbb{R}^{d}, \alpha \in \mathbb{N}_{0}^{d} .
$$

(c) $f \in \mathcal{S}\left(\mathbb{R}^{d}\right)$ and

$$
\lim _{n \rightarrow+\infty}\left(\max _{|\alpha|=n}\left\|f^{(\alpha)}(x)\right\|_{L^{p}}\right)^{1 / n}=R_{\hat{f}} \leq R .
$$

(d) $f \in \mathcal{S}\left(\mathbb{R}^{d}\right)$ and supp $\hat{f} \subseteq Q_{R}$. 
(e) $f \in \mathcal{S}\left(\mathbb{R}^{d}\right)$ and

$$
\lim _{n \rightarrow+\infty}\left\||\xi|_{\infty}^{n} \operatorname{Wig} f(x, \xi)\right\|_{L^{p, q}}^{1 / n}=R_{\hat{f}} \leq R
$$

Proof. It follows directly from Theorem 3.17 with $\omega(t)=\log (1+t)$ and the observation that (3.41) and (3.42) can be required just for $\lambda=0$ since we have $f \in \mathcal{S}\left(\mathbb{R}^{d}\right)$.

Note also that we can substitute $e^{\lambda \omega\left(\frac{x}{n+1}\right)}$ with $\frac{\left(1+\left.|x|\right|^{\lambda}\right.}{(n+1)^{\lambda}}$ instead of $\left(1+\frac{|x|}{n+1}\right)^{\lambda}$ since

$$
\left(\frac{1+|x|}{n+1}\right)^{\lambda} \leq\left(1+\frac{|x|}{n+1}\right)^{\lambda} \leq\left(1+\frac{1+|x|}{n+1}\right)^{[\lambda]+1}=\sum_{k=0}^{[\lambda]+1}\left(\begin{array}{c}
{[\lambda]+1} \\
k
\end{array}\right)\left(\frac{1+|x|}{n+1}\right)^{k} .
$$

Example 3.20. For $k \in \mathbb{N}_{0}$, let $e_{k}$ be the Hermite function on $\mathbb{R}$ defined by

$$
e_{k}(x)=\frac{1}{\left(2^{k} k ! \sqrt{\pi}\right)^{1 / 2}} e^{-x^{2} / 2} H_{k}(x), \quad x \in \mathbb{R},
$$

where the Hermite polynomial $H_{k}(x)$ of degree $k$ is given by

$$
H_{k}(x)=(-1)^{k} e^{x^{2}} \frac{d^{k}}{d x^{k}} e^{-x^{2}}, \quad x \in \mathbb{R} .
$$

The Hermite functions $e_{k} \in \mathcal{S}_{\omega}(\mathbb{R})$ (see [23, Lemma 3.2] and [11, Remark 4.17]). Then the Wigner transform $\operatorname{Wig}\left(e_{j}, e_{k}\right) \in \mathcal{S}_{\omega}\left(\mathbb{R}^{2}\right)$ and the Fourier-Wigner transform

$$
V\left(e_{j}, e_{k}\right)(y, t):=\frac{1}{2 \pi} \int_{\mathbb{R}} e_{j}\left(x+\frac{t}{2}\right) e_{k}\left(x-\frac{t}{2}\right) e^{i y x} d x
$$

is the inverse Fourier transform of $\operatorname{Wig}\left(e_{j}, e_{k}\right)$ (see [27]):

$$
\operatorname{Wig}\left(e_{j}, e_{k}\right)(x, \xi)=\mathcal{F}\left(V\left(e_{j}, e_{k}\right)\right)(x, \xi) .
$$

Let us denote by

$$
e_{j, k}(y, t)=V\left(e_{j}, e_{k}\right)(y, t), \quad j, k \in \mathbb{N}_{0} .
$$

By (3.43)

$$
\operatorname{Wig}\left(e_{j}, e_{k}\right)(x, \xi)=\hat{e}_{j, k}(x, \xi)
$$

and, by [27, Thm. 3.4], for all $j, k \in \mathbb{N}_{0}$ :

$$
L e_{j, k}(y, t)=(2 k+1) e_{j, k}(y, t),
$$

where $L$ is the twisted Laplacian defined by

$$
L:=\left(D_{y}-\frac{1}{2} t\right)^{2}+\left(D_{t}+\frac{1}{2} y\right)^{2} .
$$

Then

$$
\widehat{L e_{j, k}}=(2 k+1) \hat{e}_{j, k}
$$

and, by [11, Ex. 5.4]:

$$
\hat{L} \hat{e}_{j, k}(x, \xi)=(2 k+1) \hat{e}_{j, k}(x, \xi),
$$


where

$$
\hat{L}:=\left(\frac{1}{2} D_{\xi}+x\right)^{2}+\left(\frac{1}{2} D_{x}-\xi\right)^{2} .
$$

It is well-known that the Hermite functions are eigenfunctions of the Fourier transform:

$$
\hat{e}_{k}(\xi)=\lambda e_{k}(\xi)
$$

for some $\lambda \in \mathbb{C}$. Since $e_{k}$ does not have compact support, we have therefore that $\hat{e}_{k}$ does not have compact support, i.e. $R_{\hat{e}_{k}}=+\infty$. Since $e_{k} \in \mathcal{S}_{\omega}(\mathbb{R})$, by Corollary 3.14 we have that for all $p, q \in[1,+\infty]$ and $\mu, \lambda \geq 0$ :

$$
\lim _{n \rightarrow+\infty}\left\|e^{\lambda \omega\left(\frac{x}{n+1}\right)+\mu \omega(\xi)}|\xi|^{n} \operatorname{Wig}\left(e_{k}, e_{k}\right)(x, \xi)\right\|_{L^{p, q}}^{1 / n}=+\infty
$$

i.e. the eigenfunctions $\hat{e}_{k, k}=\operatorname{Wig}\left(e_{k}, e_{k}\right)$ of $\hat{L}$ satisfy:

$$
\lim _{n \rightarrow+\infty}\left\|e^{\lambda \omega\left(\frac{x}{n+1}\right)+\mu \omega(\xi)}|\xi|^{n} \hat{e}_{k, k}(x, \xi)\right\|_{L^{p, q}}^{1 / n}=+\infty, \quad \forall \mu, \lambda \geq 0 .
$$

Moreover, Proposition 3.3 and Remark 3.5 imply that the Hermite functions $e_{k}$ satisfy

$$
\lim _{n \rightarrow+\infty}\left\|e^{\lambda \omega\left(\frac{x}{n+1}\right)} \frac{d^{n}}{d x^{n}} e_{k}(x)\right\|_{L^{p}}^{1 / n}=+\infty
$$

for all $\lambda \geq 0$ and $p \in[1,+\infty]$.

\section{Arbitrary support}

In order to characterize the support of $\hat{f}$ in terms of the growth of some derivatives of $f$ when supp $\hat{f}$ is not compact, we replace, in the definition of $\mathrm{PW}_{R}^{\omega}\left(\mathbb{R}^{d}\right)$, the derivatives $D^{\alpha}$ by the iterates $P(D)^{n}$ of a linear partial differential operator with constant coefficients and generalize some results of [4].

Given a polynomial $P \in \mathbb{C}\left[\xi_{1}, \ldots, \xi_{d}\right]$ we denote by $P(D)$ the corresponding linear partial differential operator with symbol $P$, where we use the standard notation $D_{j}:=-i \partial_{j}$. Following [4], we define for an ultradistribution $T$ on $\mathbb{R}^{d}$ and a polynomial $P \in \mathbb{C}\left[\xi_{1}, \ldots, \xi_{d}\right]$,

$$
R(P, T):=\sup \{|P(\xi)|: \xi \in \operatorname{supp} T\},
$$

with the convention that $R(P, T)=0$ if $T \equiv 0$.

As in the previous sections, $\omega$ is a non-quasianalytic weight as in Definition 2.1, $\varphi$ is as in $(\delta)$ in Definition 2.1, and $\varphi^{*}$ is as in (2.4).

Lemma 4.1. Let $P \in \mathbb{C}\left[\xi_{1}, \ldots, \xi_{d}\right]$ be a polynomial of degree $m \geq 1$. Then, for all $k \in \mathbb{N}_{0}^{d}$ and $n \in \mathbb{N}$ :

$$
D_{\xi}^{k} P(\xi)^{n}=\sum_{\ell=0}^{|k|} \frac{n !}{(n-\ell) !} P_{\ell, k}(\xi) P(\xi)^{n-\ell},
$$

for polynomials $P_{\ell, k}(\xi)$ independent of $n$ and of degree $\operatorname{deg} P_{\ell, k} \leq \ell(m-1)$. 
Proof. Let us prove it by induction on $|k|$. If $|k|=0$ then the statement is trivial with $P_{0,0} \equiv 1$. Assume (4.2) to be valid for $|k|$, and let us prove it for $|k|+1$, i.e. for a multi-index $k+\boldsymbol{e}_{j}$ for some $1 \leq j \leq d$, where $\boldsymbol{e}_{j}$ is the vector with all entries equal to 0 except the $j$-th entry equal to 1 .

By the inductive assumption

$$
\begin{aligned}
D_{\xi}^{k+e_{j}} P(\xi)^{n} & =\sum_{\ell=0}^{|k|} \frac{n !}{(n-\ell) !} D_{\xi_{j}}\left[P_{\ell, k}(\xi) P(\xi)^{n-\ell}\right] \\
& =\sum_{\ell=0}^{|k|} \frac{n !}{(n-\ell) !}\left[D_{\xi_{j}} P_{\ell, k}(\xi) \cdot P(\xi)^{n-\ell}+P_{\ell, k}(\xi)(n-\ell) \cdot D_{\xi_{j}} P(\xi) \cdot P(\xi)^{n-\ell-1}\right] \\
& =\sum_{\ell=0}^{|k|} \frac{n !}{(n-\ell) !} D_{\xi_{j}} P_{\ell, k}(\xi) \cdot P(\xi)^{n-\ell}+\sum_{\ell=0}^{|k|} \frac{n !}{(n-\ell-1) !} P_{\ell, k}(\xi) \cdot D_{\xi_{j}} P(\xi) \cdot P(\xi)^{n-\ell-1}
\end{aligned}
$$

with $\operatorname{deg}\left(P_{\ell, k}(\xi) D_{\xi_{j}} P(\xi)\right) \leq \ell(m-1)+(m-1)=(\ell+1)(m-1)$.

We can thus write

$$
D_{\xi}^{k+\boldsymbol{e}_{j}} P(\xi)^{n}=\sum_{\tilde{\ell}=0}^{|k|+1} \frac{n !}{(n-\tilde{\ell}) !} P_{\tilde{\ell}, k+\boldsymbol{e}_{j}}(\xi) P(\xi)^{n-\tilde{\ell}},
$$

for some polynomials $P_{\tilde{\ell}, k+\boldsymbol{e}_{j}}$ not depending on $n$ and of degree $\operatorname{deg} P_{\tilde{\ell}, k+\boldsymbol{e}_{j}} \leq \tilde{\ell}(m-1)$.

Theorem 4.2. Let $P \in \mathbb{C}\left[x_{1}, \ldots, x_{d}\right]$ be a polynomial of degree $m \geq 1$. Let $f \in \mathcal{S}_{\omega}\left(\mathbb{R}^{d}\right), R>0$ and let $R(P, \hat{f})$ be defined as in (4.1). Then the following conditions are equivalent:

(a) For all $\lambda>0$ there is $C_{\lambda}>0$ such that for each $n \in \mathbb{N}_{0}$ and $x \in \mathbb{R}^{d}$ we have

$$
\left|P(D)^{n} f(x)\right| \leq C_{\lambda} R^{n} e^{-\lambda \omega\left(\left|\frac{x}{n+1}\right|^{1 / m}\right)} ;
$$

(b) $R(P, \hat{f}) \leq R$.

Proof. Let us first prove that $(a) \Rightarrow(b)$. Let $\xi_{0} \in \mathbb{R}^{d}$ and $\varepsilon>0$ such that $\left|P\left(\xi_{0}\right)\right| \geq R+\varepsilon>0$. We have to prove that $\hat{f}\left(\xi_{0}\right)=0$.

For every $\lambda>0$ and $n \in \mathbb{N}_{0}$ we have, from $(a)$ :

$$
\begin{aligned}
\left|\hat{f}\left(\xi_{0}\right)\right| & =\left|\frac{1}{P\left(\xi_{0}\right)^{n}} \int_{\mathbb{R}^{d}}\left(P(D)^{n} f(x)\right) e^{-i\left\langle\xi_{0}, x\right\rangle} d x\right| \\
& \leq \frac{1}{\left|P\left(\xi_{0}\right)\right|^{n}} \int_{\mathbb{R}^{d}} C_{\lambda} R^{n} e^{-\lambda \omega\left(\left|\frac{x}{n+1}\right|^{1 / m}\right)} d x \\
& =C_{\lambda} \frac{1}{\left|P\left(\xi_{0}\right)\right|^{n}} R^{n}(n+1)^{d} \int_{\mathbb{R}^{d}} e^{-\lambda \omega\left(|y|^{1 / m}\right)} d y \\
& =C_{m}^{\prime}\left(\frac{R}{\left|P\left(\xi_{0}\right)\right|}\right)^{n}(n+1)^{d}
\end{aligned}
$$

for some $C_{m}^{\prime}>0$, choosing $\lambda$ sufficiently large in such a way that $e^{-\lambda \omega\left(|y|^{1 / m}\right)} \in L^{1}\left(\mathbb{R}^{d}\right)$, cf. (2.3). Letting $n \rightarrow+\infty$ we have that $\hat{f}\left(\xi_{0}\right)=0$ since $\left|P\left(\xi_{0}\right)\right| \geq R+\varepsilon$. Therefore $(b)$ is satisfied. 
Conversely, let us prove that $(b) \Rightarrow(a)$. By the Fourier inversion formula, for $x \neq 0$ and $N \in \mathbb{N}_{0}$ :

$$
\begin{aligned}
\left|P(D)^{n} f(x)\right| & =\frac{1}{(2 \pi)^{d}}\left|\int_{\mathbb{R}^{d}} P(\xi)^{n} \hat{f}(\xi) e^{i\langle x, \xi\rangle} d \xi\right| \\
\leq & \frac{1}{|x|^{2 N}}\left|\int_{\mathbb{R}^{d}} P(\xi)^{n} \hat{f}(\xi) \Delta_{\xi}^{N} e^{i\langle x, \xi\rangle} d \xi\right| \\
\leq & \frac{1}{|x|^{2 N}} \int_{\mathbb{R}^{d}}\left|\Delta_{\xi}^{N}\left(P(\xi)^{n} \hat{f}(\xi)\right)\right| d \xi \\
\leq & \frac{1}{|x|^{2 N}} \sum_{|\nu|=N} \frac{N !}{\nu !} \int_{|P(\xi)| \leq R}\left|D_{\xi_{1}}^{2 \nu_{1}} \cdots D_{\xi_{d}}^{2 \nu_{d}}\left(P(\xi)^{n} \hat{f}(\xi)\right)\right| d \xi \\
\leq & \frac{1}{|x|^{2 N}} \sum_{|\nu|=N} \frac{N !}{\nu !} \sum_{k_{1}=0}^{2 \nu_{1}}\left(\begin{array}{c}
2 \nu_{1} \\
k_{1}
\end{array}\right) \cdots \sum_{k_{d}=0}^{2 \nu_{d}}\left(\begin{array}{c}
2 \nu_{d} \\
k_{d}
\end{array}\right) \\
& \cdot \int_{|P(\xi)| \leq R}\left|D_{\xi}^{k} P(\xi)^{n}\right| \cdot\left|D_{\xi}^{2 \nu-k} \hat{f}(\xi)\right| d \xi \\
\leq & \frac{1}{|x|^{2 N}} \sum_{|\nu|=N} \frac{N !}{\nu !} \sum_{k_{1}=0}^{2 \nu_{1}}\left(\begin{array}{c}
2 \nu_{1} \\
k_{1}
\end{array}\right) \cdots \sum_{k_{d}=0}^{2 \nu_{d}}\left(\begin{array}{c}
2 \nu_{d} \\
k_{d}
\end{array}\right) \sum_{\ell=0}^{|k|} \frac{n !}{(n-\ell) !} \\
& \cdot \int_{|P(\xi)| \leq R}\left|P_{\ell, k}(\xi)\right| \cdot|P(\xi)|^{n-\ell}\left|D_{\xi}^{2 \nu-k} \hat{f}(\xi)\right| d \xi
\end{aligned}
$$

for polynomials $P_{\ell, k}(\xi)$ with $\operatorname{deg} P_{\ell, k} \leq \ell(m-1)$ independent of $n$, by Lemma 4.1 .

Since $\hat{f} \in \mathcal{S}_{\omega}\left(\mathbb{R}^{d}\right)$ we thus have that for every $\mu, \lambda>0$ there exists $C_{\mu, \lambda}>0$ such that

$$
\begin{aligned}
\left|P(D)^{n} f(x)\right| \leq & \sum_{|\nu|=N} \frac{N !}{\nu !} \sum_{k_{1}=0}^{2 \nu_{1}}\left(\begin{array}{c}
2 \nu_{1} \\
k_{1}
\end{array}\right) \cdots \sum_{k_{d}=0}^{2 \nu_{d}}\left(\begin{array}{c}
2 \nu_{d} \\
k_{d}
\end{array}\right) \sum_{\ell=0}^{|k|} n^{\ell} \frac{1}{|x|^{2 N}} R^{n-|k|} \\
& \cdot \int_{|P(\xi)| \leq R}\left|P_{\ell, k}(\xi)\right| \cdot|P(\xi)|^{|k|-\ell} \cdot\left|D_{\xi}^{2 \nu-k} \hat{f}(\xi)\right| d \xi \\
\leq & \sum_{|\nu|=N} \frac{N !}{\nu !} \sum_{k_{1}=0}^{2 \nu_{1}}\left(\begin{array}{c}
2 \nu_{1} \\
k_{1}
\end{array}\right) \cdots \sum_{k_{d}=0}^{2 \nu_{d}}\left(\begin{array}{c}
2 \nu_{d} \\
k_{d}
\end{array}\right) \sum_{\ell=0}^{|k|} n^{|k|} \frac{1}{|x|^{2 N}} R^{n}\left(1+\frac{1}{R}\right)^{2 N} \\
& \cdot \int_{|P(\xi)| \leq R}(1+|\xi|)^{d+1}\left|P_{\ell, k}(\xi)\right| \cdot|P(\xi)|^{|k|-\ell} \cdot\left|D_{\xi}^{2 \nu-k} \hat{f}(\xi)\right|(1+|\xi|)^{-(d+1)} d \xi \\
\leq & \sum_{|\nu|=N} \frac{N !}{\nu !} \sum_{k_{1}=0}^{2 \nu_{1}}\left(\begin{array}{c}
2 \nu_{1} \\
k_{1}
\end{array}\right) \cdots \sum_{k_{d}=0}^{2 \nu_{d}}\left(\begin{array}{c}
2 \nu_{d} \\
k_{d}
\end{array}\right) \sum_{\ell=0}^{|k|} n^{2 N} \frac{1}{|x|^{2 N}} R^{n}\left(1+\frac{1}{R}\right)^{2 N} \\
& \cdot C_{\mu, \lambda} e^{\lambda \varphi^{*}}\left(\frac{2 N-|k|}{\lambda}\right) e^{\mu \varphi^{*}\left(\frac{m|k|-\ell+d+1}{\mu}\right)} \int_{\mathbb{R}^{d}} \frac{1}{(1+|\xi|)^{d+1}} d \xi,
\end{aligned}
$$

by Theorem $2.4(\mathrm{e})$. 
Now, since $\varphi^{*}$ is increasing, we have that

$$
e^{\lambda \varphi^{*}\left(\frac{2 N-|k|}{\lambda}\right)} \leq e^{\lambda \varphi^{*}\left(\frac{2 m N-m|k|}{\lambda}\right)}
$$

and

$$
e^{\mu \varphi^{*}\left(\frac{m|k|-\ell+d+1}{\mu}\right)} \leq C_{\mu} e^{\frac{\mu}{2} \varphi^{*}\left(\frac{m|k|-\ell}{\mu / 2}\right)} e^{\frac{\mu}{2} \varphi^{*}\left(\frac{d+1}{\mu / 2}\right)} \leq C_{\mu, d} e^{\frac{\mu}{2} \varphi^{*}\left(\frac{m|k|}{\mu / 2}\right)}
$$

by Lemma 4.6(ix).

Moreover, taking $\lambda=\mu / 2$, we have that

$$
e^{\lambda \varphi^{*}\left(\frac{2 m N-m|k|}{\lambda}\right)} e^{\lambda \varphi^{*}\left(\frac{m|k|}{\lambda}\right)} \leq e^{\lambda \varphi^{*}\left(\frac{2 m N}{\lambda}\right)}
$$

by Lemma $4.6($ ii).

We use now (4.5), (4.6) and (4.7) in (4.4) to obtain that for every $\lambda>0$ there exists $C_{\lambda}>0$ such that

$$
\begin{aligned}
\left|P(D)^{n} f(x)\right| \leq & \sum_{|\nu|=N} \frac{N !}{\nu !} \sum_{k_{1}=0}^{2 \nu_{1}}\left(\begin{array}{c}
2 \nu_{1} \\
k_{1}
\end{array}\right) \cdots \sum_{k_{d}=0}^{2 \nu_{d}}\left(\begin{array}{c}
2 \nu_{d} \\
k_{d}
\end{array}\right)(2 N+1) n^{2 N} \\
& \cdot \frac{1}{|x|^{2 N}} R^{n}\left(1+\frac{1}{R}\right)^{2 N} C_{\lambda} e^{\lambda \varphi^{*}\left(\frac{2 m N}{\lambda}\right)} \\
\leq & C_{\lambda} R^{n} \frac{1}{|x|^{2 N}} d^{N} 2^{2 N} 2^{2 N} n^{2 N}\left(1+\frac{1}{R}\right)^{2 N} e^{\lambda \varphi^{*}\left(\frac{2 m N}{\lambda}\right)} \\
\leq & C_{\lambda} R^{n}\left[\frac{1}{|x|^{2 N / m}} d^{2 N} 4^{2 N}(n+1)^{\frac{2 N}{m}}\left(1+\frac{1}{R}\right)^{2 N} e^{\frac{\lambda}{m} \varphi^{*}\left(\frac{2 N}{\lambda / m}\right)}\right]^{m} .
\end{aligned}
$$

Taking the infimum over $N \in \mathbb{N}_{0}$ and applying Lemma 4.6(vi), we have that for all $|x| \geq$ $(4 d)^{m}(n+1)\left(1+\frac{1}{R}\right)^{m}$

$$
\left|P(D)^{n} f(x)\right| \leq C_{\lambda} R^{n}\left[e^{-\left(\frac{\lambda}{m}-\frac{2}{b}\right) \omega\left(\frac{|x|^{1 / m}}{4 d(n+1)^{1 / m}(1+1 / R)}\right)-\frac{2 a}{b}}\right]^{m},
$$

for $a \in \mathbb{R}, b>0$ as in condition $(\gamma)$ of Definition 2.1. For $|x|<(4 d)^{m}(n+1)\left(1+\frac{1}{R}\right)^{m}$ we have

$$
\begin{aligned}
\left|P(D)^{n} f(x)\right| & =\frac{1}{(2 \pi)^{d}}\left|\int_{\mathbb{R}^{d}} P(\xi)^{n} \hat{f}(\xi) e^{i\langle x, \xi\rangle} d \xi\right| \\
& \leq \int_{\operatorname{supp} \hat{f}}|P(\xi)|^{n}|\hat{f}(\xi)| d \xi \leq C R^{n},
\end{aligned}
$$

with $C=\|\hat{f}\|_{L^{1}\left(\mathbb{R}^{d}\right)}$ (observe that $C$ is finite since $\hat{f} \in \mathcal{S}_{\omega}\left(\mathbb{R}^{d}\right)$ ). Since $\omega$ is increasing, we then have that $(4.8)$ is satisfied for $|x|<(4 d)^{m}(n+1)\left(1+\frac{1}{R}\right)^{m}$ with $C_{\lambda}=C e^{\left|\lambda-\frac{2 m}{b}\right| \omega(1)+\frac{2 m a}{b}}$, and so (4.8) is satisfied for every $x \in \mathbb{R}^{d}$. From (2.2) we finally have that for every $\mu>0$ there exists $C_{\mu}>0$, depending on $\mu, m, a, b, d$ and $R$, such that

$$
\left|P(D)^{n} f(x)\right| \leq C_{\mu} R^{n} e^{-\mu \omega\left(\left|\frac{x}{n+1}\right|^{1 / m}\right)}, \quad \forall x \in \mathbb{R}^{d},
$$

i.e. (4.3) is satisfied. 
Based in some known results of Andersen [4], we can deduce easily the following corollary:

Corollary 4.3. If $P \in \mathbb{C}\left[x_{1}, \ldots, x_{d}\right]$ is a polynomial of degree $m \geq 1, f \in \mathcal{S}_{\omega}\left(\mathbb{R}^{d}\right)$ and $1 \leq p \leq$ $\infty$, we have, for all $\lambda \geq 0$,

$$
\lim _{n \rightarrow+\infty}\left\|e^{\lambda \omega\left(\left|\frac{x}{n+1}\right|^{1 / m}\right)} P(D)^{n} f(x)\right\|_{L^{p}}^{1 / n}=R(P, \hat{f}) .
$$

Proof. On one hand, from [4, Proposition 2.4], it is obvious that

$$
\liminf _{n \rightarrow+\infty}\left\|e^{\lambda \omega\left(\left|\frac{x}{n+1}\right|^{1 / m}\right)} P(D)^{n} f(x)\right\|_{L^{p}}^{1 / n} \geq R(P, \hat{f}),
$$

for all $\lambda \geq 0$. Hence, it is sufficient to prove that

$$
\limsup _{n \rightarrow+\infty}\left\|e^{\lambda \omega\left(\left|\frac{x}{n+1}\right|^{1 / m}\right)} P(D)^{n} f(x)\right\|_{L^{p}}^{1 / n} \leq R(P, \hat{f}),
$$

for any $\lambda \geq 0$. To see this we fix $\lambda \geq 0$ and consider $\mu>0$ big enough such that

$$
\left\|e^{-\mu \omega\left(|x|^{1 / m}\right)}\right\|_{L^{p}}<+\infty .
$$

Now, we assume that $R(P, \hat{f})<+\infty$. By Theorem 4.2 , for every $R \geq R(P, \hat{f})$ and every $n \in \mathbb{N}$, we have

$$
\begin{aligned}
& \left\|e^{\lambda \omega\left(\left|\frac{x}{n+1}\right|^{1 / m}\right)} P(D)^{n} f(x)\right\|_{L^{p}} \\
& \quad \leq\left\|e^{-\mu \omega\left(\left|\frac{x}{n+1}\right|^{1 / m}\right)}\right\|_{L^{p}}\left\|e^{(\lambda+\mu) \omega\left(\left|\frac{x}{n+1}\right|^{1 / m}\right)} P(D)^{n} f(x)\right\|_{L^{\infty}} \\
& \quad \leq(n+1)^{d / p} C_{\lambda+\mu}\left\|e^{-\mu \omega\left(|x|^{1 / m}\right)}\right\|_{L^{p}} R^{n} .
\end{aligned}
$$

We deduce that

$$
\limsup _{n \rightarrow+\infty}\left\|e^{\lambda \omega\left(\left|\frac{x}{n+1}\right|^{1 / m}\right)} P(D)^{n} f(x)\right\|_{L^{p}}^{1 / n} \leq R,
$$

for each $R \geq R(P, \hat{f})$, which concludes the proof.

Remark 4.4. Let us remark that Theorem 4.2 gives an estimate, in terms of $R$, of the upper bound of $|P(\xi)|$ for $\xi \in \operatorname{supp} \hat{f}$. This is interesting because $\left\{\xi \in \mathbb{R}^{d}:|P(\xi)| \leq R\right\}$ can be noncompact, so that we have some estimate on the support of $\hat{f}$ for $f \in \mathcal{S}_{\omega}\left(\mathbb{R}^{d}\right)$, with arbitrary support of $\hat{f}$. Our results should be compared with [22]. See also $[8,9,10]$.

Example 4.5. Let $P \in \mathbb{C}\left[\xi_{1}, \ldots, \xi_{d}\right]$ be a polynomial of degree $m \geq 1$. If $P$ is hypoelliptic, then

$$
V_{R}:=\left\{\xi \in \mathbb{R}^{d}:|P(\xi)| \leq R\right\}
$$

is compact. 
Indeed, if $P$ is hypoelliptic then there exist $c>0$ and $0<\sigma \leq m$ such that

$$
|P(\xi)| \geq c|\xi|^{\sigma}, \quad \forall \xi \in \mathbb{R}^{d},|\xi| \gg 1 .
$$

Therefore there exists $M>0$ such that

$$
V_{R} \subseteq\left\{\xi \in \mathbb{R}^{d}:|\xi| \leq M\right\} \cup\left\{\xi \in \mathbb{R}^{d}: c|\xi|^{\sigma} \leq|P(\xi)| \leq R\right\}
$$

and therefore is bounded and hence compact, since its trivially closed.

On the contrary, the fact that $V_{R}$ is compact does not imply that $P$ is hypoelliptic. Take, for instance,

$$
P(z)=z_{1}^{2}-z_{2}^{2}+i z_{2}, \quad z_{1}, z_{2} \in \mathbb{C}
$$

In this case

$$
V_{R}=\left\{\xi \in \mathbb{R}^{2}:\left|\xi_{1}^{2}-\xi_{2}^{2}+i \xi_{2}\right| \leq R\right\}
$$

is compact since $|P(\xi)| \leq R$ implies

$$
\begin{aligned}
& |\operatorname{Im} P(\xi)|=\left|\xi_{2}\right| \leq R \\
& |\operatorname{Re} P(\xi)|=\left|\xi_{1}^{2}-\xi_{2}^{2}\right| \leq R \Rightarrow\left|\xi_{1}\right| \leq \sqrt{\xi_{2}^{2}+R} \leq \sqrt{R^{2}+R} .
\end{aligned}
$$

However, $P(\xi)$ is not hypoelliptic since the following necessary and sufficient condition for hypoellipticity (see [25, Prop. 2.2.1]) is not satisfied:

$$
\lim _{\substack{\zeta \in V \\|\zeta| \rightarrow+\infty}}|\operatorname{Im} \zeta|=+\infty
$$

for

$$
\begin{aligned}
V & :=\left\{z \in \mathbb{C}^{2}: P(z)=0\right\} \\
& =\left\{z \in \mathbb{C}^{2}: z_{2}^{2}-i z_{2}-z_{1}^{2}=0\right\} \\
& =\left\{z \in \mathbb{C}^{2}: z_{2}=\frac{i \pm \sqrt{-1+4 z_{1}^{2}}}{2}\right\}
\end{aligned}
$$

where $\pm \sqrt{-1+4 z_{1}^{2}}$ denote the two complex roots of $4 z_{1}^{2}-1$.

Taking, for instance,

$$
\xi=\left(\xi_{1}, \frac{i+\sqrt{4 \xi_{1}^{2}-1}}{2}\right) \in V, \quad \text { for } \xi_{1} \in \mathbb{R},
$$

we have that $|\xi| \rightarrow+\infty$ for $\left|\xi_{1}\right| \rightarrow+\infty$, but

$$
|\operatorname{Im} \xi|=\left|\left(0, \frac{1}{2}\right)\right|=\frac{1}{2} .
$$




\section{Appendix}

We use the next lemma throughout the paper. Some points are elementary ((i), (ii), (iv) and (ix)), and the proofs of others can be found in the literature (for (v) and (vi) see [11, Lemma 4.7] or [15, Lemma 1.4]), but we include a full proof for the convenience of the reader. From [21] we know that for any convex and continuous function $\varphi:[0,+\infty) \rightarrow[0,+\infty)$ we can define its Young conjugate (Legendre transform in [21, Definition 1.3.2]) $\varphi^{*}$ as in (2.4) which is also convex and satisfies $\left(\varphi^{*}\right)^{*}=\varphi$.

Lemma 4.6. Let $\omega:[0,+\infty) \rightarrow[0,+\infty)$ be a continuous increasing function such that $\varphi$ : $[0,+\infty) \rightarrow[0,+\infty), \varphi(t):=\omega\left(e^{t}\right)$ is convex. Then the following properties hold:

(i) $\varphi^{*}(s) / s$ is increasing.

(ii) $\varphi^{*}(t)+\varphi^{*}(s) \leq \varphi^{*}(t+s)$, for all $t, s \geq 0$.

(iii) If there exist $\bar{A} \geq 0$ and $B \geq 1$ such that $\omega($ et $) \leq A+B \omega(t)$ for all $t \geq 0$, then for all $\lambda>0$ and $j, n \in \mathbb{N}_{0}=\mathbb{N} \cup\{0\}:$

$$
\lambda \varphi^{*}\left(\frac{j}{\lambda}\right)+n j \leq \frac{\lambda}{B^{n}} \varphi^{*}\left(\frac{j}{\lambda / B^{n}}\right)+\lambda n \frac{A}{B} .
$$

If $\omega$ is subadditive in the sense of (3.9), then we can take $A=0$ and $B=3$.

(iv) If there exist $A \geq 0$ and $B \geq 1$ such that $\omega($ et $) \leq A+B \omega(t)$ for all $t \geq 0$, then for all $\rho \geq 1, \lambda>0$ and $j \in \mathbb{N}_{0}$ :

$$
\rho^{j} e^{\lambda \varphi^{*}\left(\frac{j}{\lambda}\right)} \leq \Lambda_{\rho, \lambda} e^{\lambda^{\prime} \varphi^{*}\left(\frac{j}{\lambda^{\prime}}\right)}
$$

for all $0<\lambda^{\prime} \leq \lambda / B^{[\log \rho+1]}$ and $\Lambda_{\rho, \lambda}=e^{\lambda \frac{A}{B}[\log \rho+1]}$, where $[x]$ denotes the integer part of $x$.

(v) For all $\lambda>0$ and $k \in \mathbb{N}_{0}$ :

$$
\begin{aligned}
& t^{k} e^{-\lambda \varphi^{*}\left(\frac{k}{\lambda}\right)} \leq e^{\lambda \omega(t)}, \quad t \geq 1, \\
& t^{k} e^{-\lambda \varphi^{*}\left(\frac{k}{\lambda}\right)} \leq e^{-\lambda \varphi^{*}(0)} e^{\lambda \omega(t)}, \quad 0 \leq t \leq 1 .
\end{aligned}
$$

(vi) If there exist $a \in \mathbb{R}$ and $b>0$ such that $\omega(t) \geq a+b \log (1+t)$ for all $t \geq 0$, then for all $\sigma, \lambda>0$ and $t \geq 1$ :

$$
\inf _{j \in \mathbb{N}_{0}} t^{-\sigma j} e^{\lambda \varphi^{*}\left(\frac{\sigma j}{\lambda}\right)} \leq e^{-\left(\lambda-\frac{\sigma}{b}\right) \omega(t)-a \frac{\sigma}{b}}
$$

(vii) If $\omega(t)=o(t)$ as $t \rightarrow+\infty$, then for every $\ell \in \mathbb{N}$ there exists a constant $C_{\ell}>0$ such that

$$
s \log s \leq s+\ell \varphi^{*}\left(\frac{s}{\ell}\right)+C_{\ell}, \quad s>0 .
$$

(viii) Assume that there exist $A \geq 0$ and $B \geq 1$ such that $\omega($ et $) \leq A+B \omega(t)$ for all $t \geq 0$, and moreover $\omega(t)=o(t)$ as $t \rightarrow+\infty$. Then, for all $D, \lambda>0$, there is $C_{D, \lambda}>0$ such that for all $n \in \mathbb{N}_{0}$ :

$$
D^{n} n ! \leq C_{D, \lambda} e^{\lambda \varphi^{*}\left(\frac{n}{\lambda}\right)} .
$$

(ix) For all $j, h, r \in \mathbb{N}_{0}$ and $\lambda>0$ :

$$
e^{\lambda \varphi^{*}\left(\frac{j}{\lambda}\right)} e^{\lambda \varphi^{*}\left(\frac{r+h}{\lambda}\right)} \leq e^{\frac{\lambda}{2} \varphi^{*}\left(\frac{j+h}{\lambda / 2}\right)} e^{\frac{\lambda}{2} \varphi^{*}\left(\frac{r}{\lambda / 2}\right)} .
$$


Proof. ( $i$ ): Let us first remark that $\varphi(t) \geq 0$ (for $t \geq 0$ ) implies

$$
\varphi^{*}(0)=\sup _{t \geq 0}\{0 \cdot t-\varphi(t)\} \leq 0 .
$$

Then, by the convexity of $\varphi^{*}$, for $0<s_{1}<s_{2}$ :

$$
\varphi^{*}\left(s_{1}\right)=\varphi^{*}\left(\frac{s_{1}}{s_{2}} \cdot s_{2}+\left(1-\frac{s_{1}}{s_{2}}\right) \cdot 0\right) \leq \frac{s_{1}}{s_{2}} \varphi^{*}\left(s_{2}\right)+\left(1-\frac{s_{1}}{s_{2}}\right) \varphi^{*}(0) \leq \frac{s_{1}}{s_{2}} \varphi^{*}\left(s_{2}\right) .
$$

(ii): Since $\varphi^{*}$ is convex and $\varphi^{*}(0) \leq 0$ (see the proof of (i)), for all $t, s>0$ :

$$
\begin{aligned}
\varphi^{*}(t)+\varphi^{*}(s) & =\varphi^{*}\left((t+s) \cdot \frac{t}{t+s}+0 \cdot \frac{s}{t+s}\right)+\varphi^{*}\left((t+s) \cdot \frac{s}{t+s}+0 \cdot \frac{t}{t+s}\right) \\
& \leq \varphi^{*}(t+s) .
\end{aligned}
$$

The inequality is trivial for $t$ and/or $s$ equal to 0 .

(iii): For all $s \geq 0$,

$$
\begin{aligned}
\varphi^{*}(s) & =\sup _{t \geq 0}\{t s-\varphi(t)\} \geq \sup _{t \geq 1}\{t s-\varphi(t)\}=\sup _{\sigma \geq 0}\{(\sigma+1) s-\varphi(\sigma+1)\} \\
& =s+\sup _{\sigma \geq 0}\left\{\sigma s-\omega\left(e e^{\sigma}\right)\right\} \geq s+\sup _{\sigma \geq 0}\left\{\sigma s-A-B \omega\left(e^{\sigma}\right)\right\} \\
& =s-A+B \sup _{\sigma \geq 0}\left\{\sigma \frac{s}{B}-\varphi(\sigma)\right\}=s-A+B \varphi^{*}\left(\frac{s}{B}\right) .
\end{aligned}
$$

Iterating the procedure we easily obtain, for all $n \in \mathbb{N}$,

$$
\varphi^{*}(s) \geq B^{n} \varphi^{*}\left(\frac{s}{B^{n}}\right)+n s-A \sum_{k=0}^{n-1} B^{k} .
$$

For $s:=j \frac{B^{n}}{\lambda}$, multiplying by $\lambda / B^{n}$, we obtain

$$
\frac{\lambda}{B^{n}} \varphi^{*}\left(\frac{j}{\lambda / B^{n}}\right) \geq \lambda \varphi^{*}\left(\frac{j}{\lambda}\right)+n j-\lambda \frac{A}{B} \sum_{k=0}^{n-1} B^{-k}
$$

and hence, since $B \geq 1$,

$$
\lambda \varphi^{*}\left(\frac{j}{\lambda}\right)+n j \leq \frac{\lambda}{B^{n}} \varphi^{*}\left(\frac{j}{\lambda / B^{n}}\right)+\lambda n \frac{A}{B} .
$$

Observe that when $\omega$ is subadditive $\omega($ et $) \leq \omega(3 t) \leq 3 \omega(t)$ for every $t \geq 0$.

(iv): From (iii) we have

$$
\rho^{j} e^{\lambda \varphi^{*}\left(\frac{j}{\lambda}\right)} \leq e^{\frac{\lambda}{B^{n}} \varphi^{*}\left(\frac{j}{\lambda / B^{n}}\right)+\lambda n \frac{A}{B}-n j+j \log \rho},
$$

and therefore, for $n_{\rho}:=[\log \rho+1] \geq \log \rho$ :

$$
\rho^{j} e^{\lambda \varphi^{*}\left(\frac{j}{\lambda}\right)} \leq e^{\lambda n_{\rho} \frac{A}{B}} e^{\frac{\lambda}{B^{n} \rho} \varphi^{*}\left(\frac{j}{\lambda / B^{n} \rho}\right)} \leq e^{\lambda n_{\rho} \frac{A}{B}} e^{\lambda^{\prime} \varphi^{*}\left(\frac{j}{\lambda^{\prime}}\right)},
$$

for all $0<\lambda^{\prime} \leq \lambda / B^{n_{\rho}}$ (here, we use $(i)$ again).

$(v)$ : For all $\lambda>0, t \geq 1$ and $k \in \mathbb{N}_{0}$ :

$$
k \log t-\lambda \omega(t) \leq \sup _{t \geq 1}\{k \log t-\lambda \omega(t)\}=\lambda \sup _{s \geq 0}\left\{\frac{k}{\lambda} s-\varphi(s)\right\}=\lambda \varphi^{*}\left(\frac{k}{\lambda}\right) .
$$


Therefore $t^{k} e^{-\lambda \omega(t)} \leq e^{\lambda \varphi^{*}\left(\frac{k}{\lambda}\right)}$ for all $t \geq 1$.

On the other hand, for $0 \leq t \leq 1$ we have

$$
t^{k} e^{-\lambda \varphi^{*}\left(\frac{k}{\lambda}\right)} \leq e^{-\lambda \varphi^{*}\left(\frac{k}{\lambda}\right)} \leq e^{-\lambda \varphi^{*}(0)} e^{\lambda \omega(t)},
$$

since $\varphi^{*}$ is obviously increasing, as it is deduced directly from the definition of Young conjugate.

(vi): For all $s \geq 0$ and $\lambda, \sigma>0$ there exists $j \in \mathbb{N}_{0}$ such that $j \leq \frac{s \lambda}{\sigma}<j+1$. Then for all $t \geq 1$ :

$$
\begin{aligned}
\sup _{j \in \mathbb{N}_{0}}\left\{\sigma j \log t-\lambda \varphi^{*}\left(\frac{\sigma j}{\lambda}\right)\right\} & =\lambda \sup _{j \in \mathbb{N}_{0}}\left\{\frac{\sigma j+\sigma}{\lambda} \log t-\varphi^{*}\left(\frac{\sigma j}{\lambda}\right)\right\}-\sigma \log t \\
& \geq \lambda \sup _{s \geq 0}\left\{s \log t-\varphi^{*}(s)\right\}-\sigma\left(\frac{1}{b} \omega(t)-\frac{a}{b}\right) \\
& =\lambda \varphi^{* *}(\log t)-\frac{\sigma}{b} \omega(t)+a \frac{\sigma}{b}=\left(\lambda-\frac{\sigma}{b}\right) \omega(t)+a \frac{\sigma}{b},
\end{aligned}
$$

and hence

$$
\inf _{j \in \mathbb{N}_{0}} t^{-\sigma j} e^{\lambda \varphi^{*}\left(\frac{\sigma j}{\lambda}\right)} \leq e^{-\left(\lambda-\frac{\sigma}{b}\right) \omega(t)-a \frac{\sigma}{b}} .
$$

(vii): Since $\omega(t)=o(t)$ as $t \rightarrow+\infty$, for every $\ell$ there exists $R_{\ell}>0$ such that

$$
\omega(t) \leq \frac{1}{\ell} t+R_{\ell}, \quad t \geq 0
$$

Then, for all $s \geq 1$, we have

$$
\varphi^{*}\left(\frac{s}{\ell}\right)=\sup _{t \geq 0}\left\{t \frac{s}{\ell}-\omega\left(e^{t}\right)\right\} \geq \sup _{t \geq 0}\left\{t \frac{s}{\ell}-\frac{1}{\ell} e^{t}\right\}-R_{\ell}=\frac{s}{\ell} \log s-\frac{s}{\ell}-R_{\ell} .
$$

Hence the thesis is proved for $s \geq 1$ for $C_{\ell}=\ell R_{\ell}$. For $s \in(0,1)$ the result is obvious since $s \log s<0$.

(viii): By Stirling's formula there exists $H>0$ such that

$$
n ! \leq H \sqrt{n}\left(\frac{n}{e}\right)^{n} \leq H 2^{n}\left(\frac{n}{e}\right)^{n}, \quad n \in \mathbb{N} .
$$

Therefore, from (vii) and (iv), for every $\ell \in \mathbb{N}$ there exists $C_{\ell}>0$ such that

$$
\begin{aligned}
D^{n} n ! & \leq H\left(\frac{2 D}{e}\right)^{n} n^{n} \leq H\left(\frac{2 D}{e}\right)^{n} e^{n+\ell \varphi^{*}\left(\frac{n}{\ell}\right)+C_{\ell}} \\
& =H e^{C_{\ell}}(2 D)^{n} e^{\ell \varphi^{*}\left(\frac{n}{\ell}\right)} \leq H e^{C_{\ell}} \Lambda_{2 D, \ell} e^{\lambda \varphi^{*}\left(\frac{n}{\lambda}\right)}
\end{aligned}
$$

for all $0<\lambda \leq \frac{\ell}{B^{[\log (2 D)+1]}}$ and $\Lambda_{2 D, \ell}=e^{\ell \frac{A}{B}[\log (2 D)+1]}$, provided $D \geq 1 / 2$.

Therefore, for every $\lambda>0$, we can choose a natural number $\ell \geq \lambda B^{[\log (2 D)+1]}$ to obtain

$$
D^{n} n ! \leq C_{D, \lambda} e^{\lambda \varphi^{*}\left(\frac{n}{\lambda}\right)},
$$

for $C_{D, \lambda}=H e^{C_{\ell}} \Lambda_{2 D, \ell}$.

(ix): From (ii) and the convexity of $\varphi^{*}$ :

$$
e^{\lambda \varphi^{*}\left(\frac{j}{\lambda}\right)} e^{\lambda \varphi^{*}\left(\frac{r+h}{\lambda}\right)} \leq e^{\lambda \varphi^{*}\left(\frac{j+r+h}{\lambda}\right)}=e^{\lambda \varphi^{*}\left(\frac{1}{2} \frac{j+h}{\lambda / 2}+\frac{1}{2} \frac{r}{\lambda / 2}\right)} \leq e^{\frac{\lambda}{2} \varphi^{*}\left(\frac{j+h}{\lambda / 2}\right)} e^{\frac{\lambda}{2} \varphi^{*}\left(\frac{r}{\lambda / 2}\right)} .
$$


Acknowledgments. The authors are grateful for the careful reading and the suggestions of the referee, that improved the paper. The authors were partially supported by the INdAMGnampa Project 2017 "Equazioni a Derivate Parziali, Analisi di Gabor ed Analisi Microlocale", by the Projects FAR 2014 and FAR 2017 (University of Ferrara), by the Project FFABR 2017 (MIUR). The research of the second author was partially supported by the project MTM201676647-P.

\section{References}

[1] A. Albanese, D. Jornet, A. Oliaro, Quasianalytic wave front sets for solutions of linear partial differential operators, Integr. Equ. Oper. Theory 66 (2010), 153-181.

[2] N. B. Andersen, Real Paley-Wiener theorems, Bull. London Math. Soc. 36, (2004), 504-508.

[3] N. B. Andersen, Entire $L^{p}$-functions of exponential type, Expo. Math. 32 (2014), 199-220.

[4] N. B. Andersen, M. De Jeu, Real Paley-Wiener theorems and local spectral radius formulas, Trans. Amer. Math. Soc. 362, 7 (2010), 3613-3640.

[5] H. H. Bang, A property of infinitely differentiable functions, Proc. Amer. Math. Soc. 108, 1 (1990), 73-76.

[6] G. Björck, Linear partial differential operators and generalized distributions, Ark. Mat. 6, n. 21 (1966), 351-407.

[7] C. Boiti, E. Gallucci, The overdetermined Cauchy problem for $\omega$-ultradifferentiable functions, Manuscripta Math. 155, n.3-4 (2018), 419-448.

[8] C. Boiti, D. Jornet, A characterization of the wave front set defined by the iterates of an operator with constant coefficients, RACSAM - Revista de la Real Academia de Ciencias Exactas, Físicas y Naturales. Serie A. Matemáticas, 111, n.3 (2017), 891-919.

[9] C. Boiti, D. Jornet, A simple proof of Kotake-Narasimhan Theorem in some classes of ultradifferentiable functions, J. Pseudo-Differ. Oper. Appl. 8(2), (2017), 297-317.

[10] C. Boiti, D. Jornet, J. Juan-Huguet, Wave front sets with respect to the iterates of an operator with constant coefficients, Abstr. Appl. Anal. 2014, Article ID 438716 (2014), 1-17.

[11] C. Boiti, D. Jornet, A. Oliaro, Regularity of partial differential operators in ultradifferentiable spaces and Wigner type transforms, J. Math. Anal. Appl. 446 (2017), 920-944.

[12] C. Boiti, D. Jornet, A. Oliaro, The Gabor wave front set in spaces of ultradifferentiable functions, Monatsh. Math. 188 (2019), no. 2, 199-246.

[13] J. Bonet, R. Meise, S.N. Melikhov, A comparison of two different ways to define classes of ultradifferentiable functions, Bull. Belg. Math. Soc. Simon Stevin 14(3) (2007), 425-444.

[14] R.W. Braun, R. Meise, B.A. Taylor, Ultradifferentiable functions and Fourier analysis, Result. Math. 17 (1990), 206-237.

[15] C. Fernández, A. Galbis, D. Jornet, Pseudodifferential operators on non-quasianalytic classes of Beurling type, Studia Math. 167 (2005), 99-131.

[16] C. Fieker, P-Konvexität und $\omega$-Hypoelliptizität für partielle Differentialoperatoren mit konstanten Koeffizienten. Diplomarbeit, Heinrich-Heine-Universität, 1993.

[17] G.B. Folland, Real Analysis. Modern Techniques and Their Applications, Second Edition, WileyInterscience, New-York (1999).

[18] K. Gröchenig, Foundations of Time-Frequency Analysis, Birkhäuser, Boston (2001).

[19] K. Gröchenig, G. Zimmermann, Spaces of test functions via the STFT, J. Funct. Spaces Appl. 2, n. 1 (2004), 25-53

[20] L. Hörmander, The Analysis of Linear Partial Differential Operators II, Springer-Verlag, Berlin, 1983.

[21] L. Hörmander, Notions of convexity, Progress in Mathematics, 127, Birkhäuser Boston, (1994).

[22] J. Juan-Huguet, A Paley-Wiener type theorem for generalized non-quasianalytic classes, Studia Math. 208 (2012), no. 1, 31-46.

[23] M. Langenbruch, Hermite functions and weighted spaces of generalized functions, Manuscripta Math. 119, n. 3 (2006), 269-285. 
[24] R. Meise, B.A. Taylor, Whitney's extension theorem for ultradifferentiable functions of Beurling type, Ark. Math. 26, n. 2 (1988), 265-287.

[25] L. Rodino, Linear partial differential operators and Gevrey spaces, World Scientific Publishing Co., Inc. River Edge, NJ, (1993).

[26] V.K. Tuan, On the supports of functions, Numer. Funct. Anal. Optim. 20 (1999), 387-394.

[27] M.-W. Wong, Weyl transforms, the heat kernel and Green functions of a degenerate elliptic operator, Ann. Global Anal. Geom., 28, n. 3 (2005), 271-283.

Dipartimento di Matematica e Informatica, Università di Ferrara, Via Machiavelli n. 30, I-44121 FERRARA, ITALY

E-mail address: chiara.boiti@unife.it

Instituto Universitario de Matemática Pura y Aplicada IUMPA, Universitat Politècnica de València, Camino de Vera, s/n, E-46071 Valencia, Spain

E-mail address: djornet@mat.upv.es

Dipartimento di Matematica, Università di Torino, Via Carlo Alberto n. 10, I-10123 Torino, ITALY

E-mail address: alessandro.oliaro@unito.it 